\title{
Comment la création d'une «bibliothèque de papyrus » à Strasbourg compensa la perte des manuscrits précieux brûlés dans le siège de 1870
}

\section{Frédéric Colin}

\section{(2) OpenEdition}

\section{Journals}

Édition électronique

URL : https://journals.openedition.org/rbnu/3314

DOI : 10.4000/rbnu.3314

ISSN : 2679-6104

Éditeur

Bibliothèque nationale et universitaire de Strasbourg

\section{Édition imprimée}

Date de publication : 1 novembre 2010

Pagination : 24-47

ISSN : 2109-2761

\section{Référence électronique}

Frédéric Colin, «Comment la création d'une «bibliothèque de papyrus » à Strasbourg compensa la perte des manuscrits précieux brûlés dans le siège de 1870 », La Revue de la BNU [En ligne], 2 | 2010, mis en ligne le 01 novembre 2010, consulté le 08 août 2021. URL : http://journals.openedition.org/ rbnu/3314; DOI : https://doi.org/10.4000/rbnu.3314

\section{(c) (i) (2)(2)}

La Revue de la BNU est mise à disposition selon les termes de la Licence Creative Commons Attribution - Pas d'Utilisation Commerciale - Partage dans les Mêmes Conditions 4.0 International. 
LE DOSSIER | Egypte-Europe, allers-retours

C

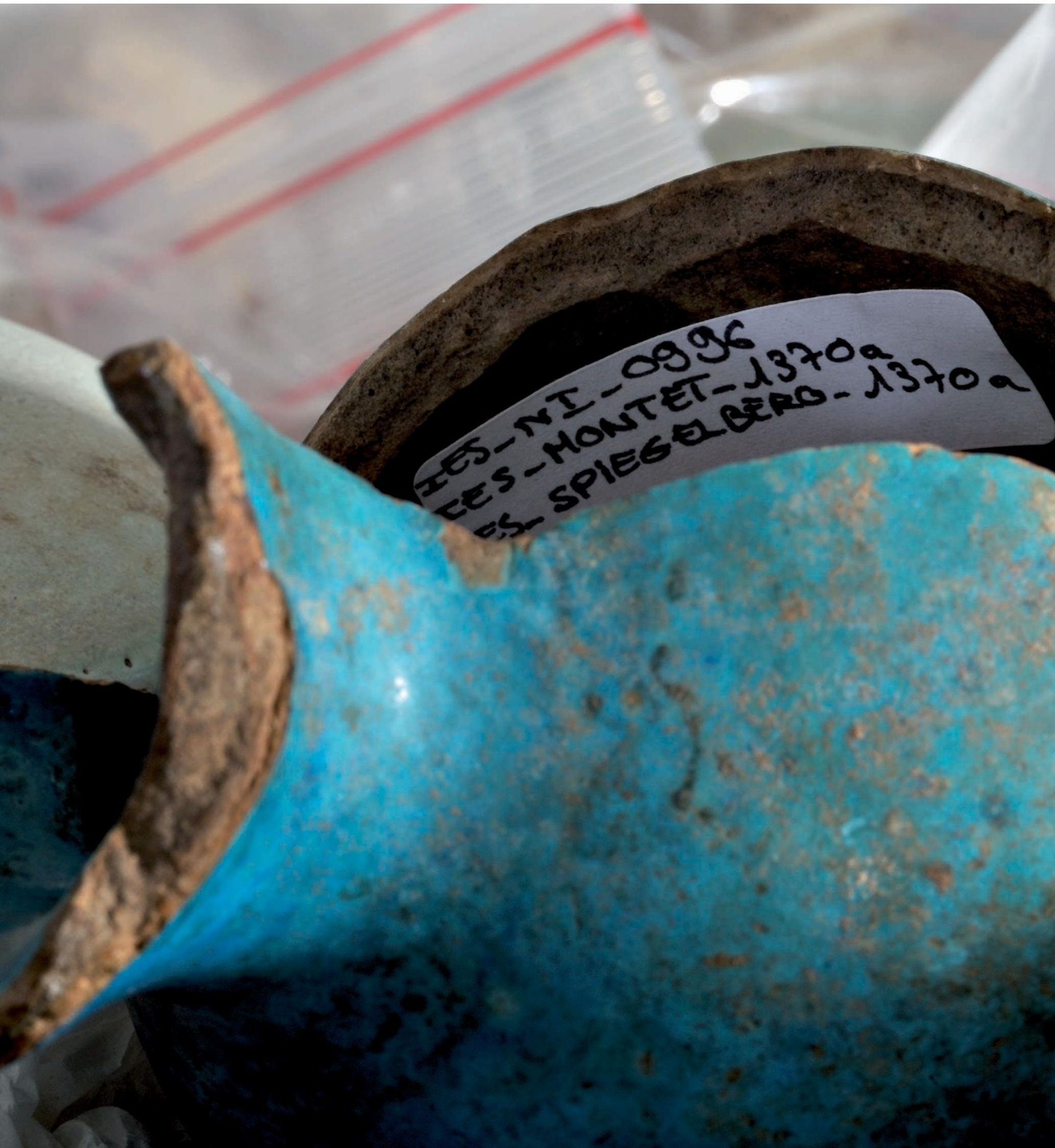




\section{Comment la création} d'une "bibliothèque de papyrus" à Strasbourg compensa la perte des manuscrits précieux brûlés dans le siège de 1870

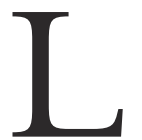

es réserves précieuses de la Bibliothèque nationale et universitaire de Strasbourg conservent un remarquable fonds de plus de 10 ooo papyrus et ostraca, qui portent des textes écrits dans la plupart des langues utilisées, de façon régulière ou anecdotique, dans l'Égypte ancienne : égyptien hiéroglyphique, hiératique, démotique et copte, grec, latin, pehlvi, araméen, hébreu et arabe ${ }^{1}$. À l'Institut d'égyptologie de l'université, d'autre part, une collection d'objets authentiques, davantage tournée vers l'archéologie et l'histoire de l'art, offre aux professeurs et aux étudiants un champ de recherche et d'expérimentation exceptionnel, car elle contient un échantillonnage de tous les types d'artefacts susceptibles de se présenter à l'archéologue sur un chantier égyptien ${ }^{2}$. Pourquoi et comment ces trésors inestimables ont-ils abouti entre les murs de ces deux institutions universitaires, parmi les dispositifs de rayonnement culturel déployés dans l'AlsaceLorraine de Guillaume II ?

Naguère, les conditions d'acquisition de la collection de papyrus de la Kaiserliche Universitäts- und Landesbibliothek (KULB, future BNU) étaient seulement connues à partir de 1902, lorsqu'un incident diplomatique interne à l'Empire allemand mit le gouvernement du Reichsland aux prises avec le ministre des Affaires étrangères $\mathrm{du}$ royaume de Prusse. Ce dernier, en effet, fit pression sur le gouverneur de l'Alsace-Lorraine en lui demandant de " conseiller " aux autorités de la bibliothèque strasbourgeoise de participer à un " cartel des papyrus allemand " (Deutsches Papyruskartell) destiné à mutualiser les moyens financiers des institutions allemandes soucieuses d'acheter des papyrus grecs en Égypte. Une étude a montré que la démarche des autorités prussiennes avait pour but de mettre un terme à la concurrence de Strasbourg, qui contrariait les projets de la commission d'acquisition de papyrus grecs littéraires des Musées royaux de Berlin en détournant une partie de l'offre et, 
craignait-on, en poussant à la hausse les prix exigés par les antiquaires ${ }^{3}$. Il est vrai que deux professeurs de l'université de Strasbourg, Wilhelm Spiegelberg (égyptologue) et Richard Reitzenstein (philologue classique) avaient récemment acquis une excellente expérience du marché des antiquités, en partant eux-mêmes à la chasse aux papyrus sur le terrain égyptien. Les résultats scientifiques de leur voyage avaient été très remarqués, comme en témoigne la parution, le $1^{\text {er }}$ décembre 1901, d'un long article du Strassburger Post consacré aux trésors papyrologiques dont ils avaient ainsi pourvu les collections de la KULB. Cependant, les circonstances de leur voyage fondateur sont restées largement inconnues jusqu'à aujourd'hui. En 1961, le papyrologue strasbourgeois Jacques Schwartz écrivait : " Malgré tous mes efforts, il m'a été impossible de retrouver, ni dans les archives de l'université ni dans ce qui subsiste des archives d'Alsace-Lorraine, aucune autre indication sur les circonstances d'achat des papyrus ${ }^{4}$. Après avoir identifié dans les Archives départementales $\mathrm{du}$ Bas-Rhin les actes relatifs à la constitution des collections égyptiennes produits par l'université impériale, j’ai pu entamer, en 2008, une recherche sur cet épisode original de l'histoire culturelle de l'Alsace, dont cet article livre au lecteur les premiers résultats.

Comme souvent dans les entreprises majeures de la science, la petite histoire académique d'un savant et la grande histoire du développement des fonds égyptiens de l'université et de la bibliothèque strasbourgeoises se tissèrent sur une seule et même trame. On ne saurait comprendre la naissance des deux collections sans retracer le parcours scientifique de W. Spiegelberg, du point de vue très prosaïque de la construction de son curriculum vitae au moment d'entrer dans la carrière.

\section{Le contexte académique de la naissance des collections égyptiennes de Strasbourg}

L'égyptologie universitaire, en Alsace, est née de la rivalité politique, militaire et culturelle qui opposait la France et l'Allemagne au lendemain de la guerre de 1870. Le 20 février 1872, le baron Franz von Roggenbach, membre du Reichstag et organisateur de la refondation de l'Université impériale de Strasbourg, invite l'égyptologue prussien Johannes Dümichen à y accepter une charge de professeur extraordinaire, pour " participer à l'œuvre d'éducation qui s'accomplit dans le pays frontalier allemand récemment conquis, et où une digne représentation de votre discipline est particulièrement nécessaire à cause de la valeur que l'on accorde aux études égyptologiques du côté français " ${ }^{5}$. C'est donc véritablement en pionnier de la science germanique sur les marges territoriales à peine conquises que Dümichen est invité à participer à une œuvre de civilisation de la population alsacienne. L'égyptologie est érigée en discipline phare, pour contrebalancer le prestige dont jouissaient les Français dans ce domaine. La création de la chaire d'égyptologie n'affichait donc pas la moindre des ambitions : concurrencer le Collège de France, dans les terres qui venaient d'échapper à ce pays.

Cette mission très valorisante attira-t-elle sur Dümichen la jalousie de certains de ses contemporains ? L'égyptologue berlinois Adolf Erman, connu pour sa plume parfois acerbe, le considérait en tout cas comme un "rêveur bizarre " et prétendait que " ses collègues strasbourgeois l'appelaient 'Dümmlichen' (petit nigaud) ${ }^{6}$. À l'occasion de l'emménagement des facultés dans le Kollegiengebäude (actuel Palais universitaire), dont la construction s'était achevée en 1884, Dümichen obtint un budget exceptionnel de 1800 marks pour fonder une collection de reproductions en plâtre achetées aux ateliers des musées de Berlin : des bustes de pharaons et des copies de reliefs de l'ancien Empire furent ainsi exposés au premier étage du Palais universitaire, dans le prolongement de l'exposition des moulages d'œuvres de l'Antiquité classique'.

La mort du savant, survenue le 7 février 1894, semble avoir plongé la Faculté de philosophie dans un certain désarroi. En effet, un jeune homme prometteur, Wilhelm Spiegelberg, était certes venu depuis Hanovre suivre les enseignements de Dümichen à Strasbourg et y avait obtenu sa Promotion le 22 novembre 1891 - ses talents naissants ne doivent pas avoir échappé à deux grandes pointures scientifiques strasbourgeoises, Adolf Michaelis (archéologue) et Theodor Nöldeke (sémitisant), qui paraissent l'avoir fait bénéficier d'une attention bienveillante dès ses débuts. Mais au décès du maître, Spiegelberg, seulement âgé de 23 ans, n'était pas encore " habilité " et ne détenait donc pas la "venia legendi ", le droit d'enseigner à l'université. En outre, contrairement à Dümichen qui avait longuement séjourné en Égypte, 


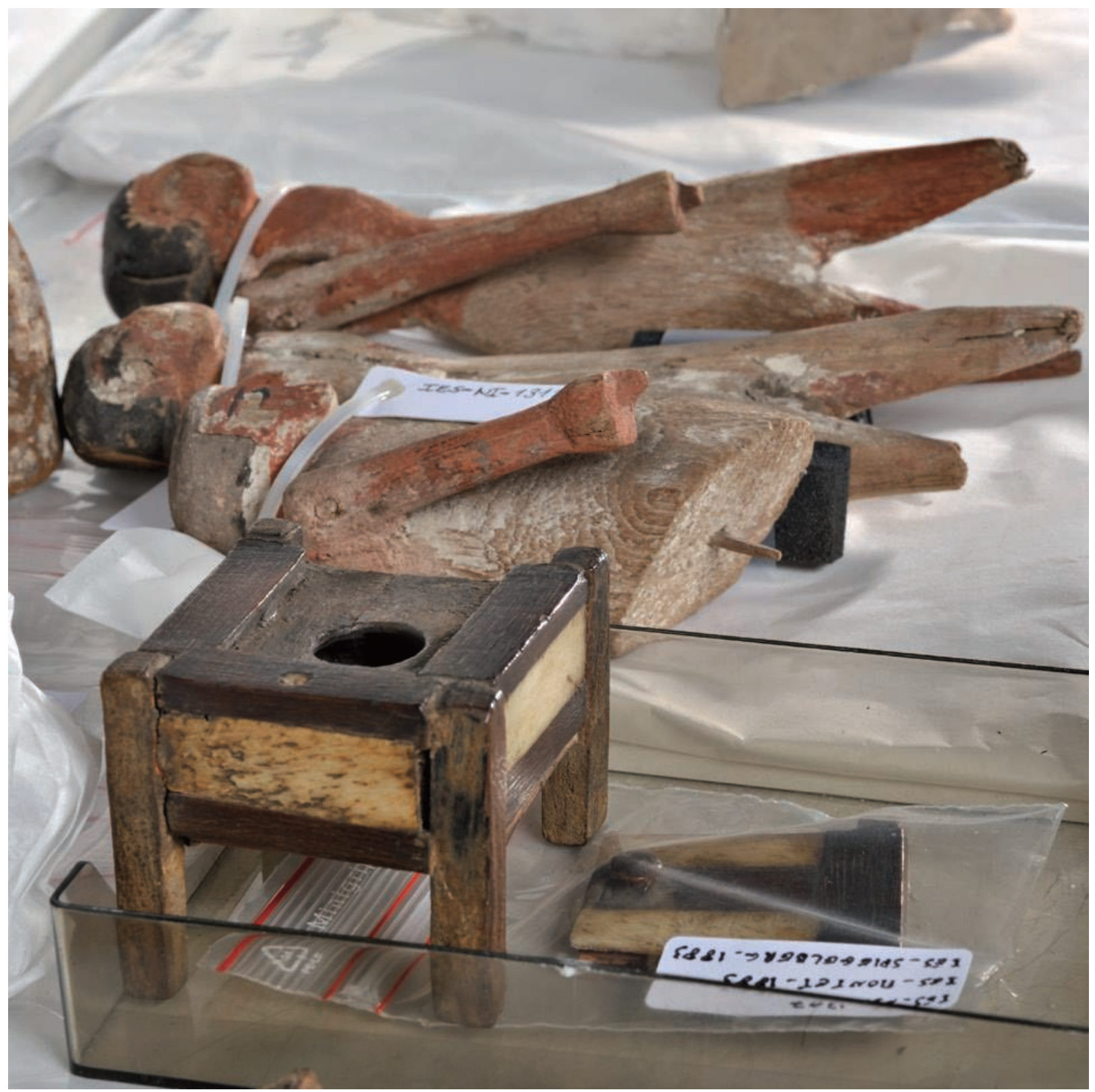

Modèles de mobilier et figurines en bois achetés en Égypte par Spiegelberg (coll. UdS) 
Spiegelberg ne s'y était encore jamais rendu et l'expérience du terrain lui faisait totalement défaut. Il était trop tôt pour songer à lui confier l'enseignement de l'égyptologie. En nous gardant de tout anachronisme, rendons à l'histoire son incertitude : en 1894, les savants de la Faculté de philosophie ignoraient que Spiegelberg serait appelé à devenir un des géants de l'égyptologie du XXe siècle. Mais ils eurent manifestement la clairvoyance de parier sur le brillant jeune homme, car la séquence rapide des actes enregistrés dans les archives de l'université, à partir du décès de Dümichen, laisse entendre que cet événement déclencha, au profit du candidat local à la succession, une entreprise bien organisée de mise en ré-

serve de la chaire d'égyptologie et de renforcement du dossier scientifique de Spiegelberg. Énumérons les grands axes de la stratégie conduite à cette fin par la Faculté, en suivant la chronologie révélée par le Journal der philosophischen Facultät ${ }^{8}$, où les doyens, en fonction pour un an, enregistraient sous la forme de brèves notices les principaux événements académiques.

1. Le 18 février 1894, l'administration par intérim de la " collection égyptologique " (c'est-àdire les moulages, la bibliothèque et le mobilier du "Seminar ") est confiée au professeur Adolf Michaelis.

2. Le 28 avril, sur proposition de la commission chargée d’organiser la succession, la Faculté décide « de ne pas faire pour l'instant de propositions de nouvelle occupation (de la chaire) " - on décide donc de ne rien décider, en laissant le temps au temps...

3. Le 21 mai, Spiegelberg dépose son dossier en vue d'engager la procédure d'habilitation, et le 27 mai le doyen de la Faculté écrit à Adolf Erman, professeur d'égyptologie à Berlin, pour lui demander d'examiner le mémoire d'habilitation du candidat. Cette première étape franchie avec succès, Spiegelberg est autorisé le 7 juillet à continuer l'épreuve, qui se clôturera le $1^{\mathrm{er}}$ août par sa leçon inaugurale. Dès cette date - moins de six mois après le décès de Dümichen, Spiegelberg est titulaire de la "venia legendi » et ha- bilité à enseigner à la Faculté de philosophie comme " Privatdozent ", c'est-à-dire comme enseignant non salarié, agrégé à l'université.

4. Il prévoit de commencer ses premières leçons lors du semestre d'été de 1895, d'après la liste des enseignements semestriels publiée par l'université. La participation régulière aux programmes de cours est en effet un des socles qui renforcent l'existence sociale des universitaires au sein d'une faculté.

5. Mais Spiegelberg est toujours un égyptologue de bibliothèque, il s'agit désormais de lui donner la carrure d'un homme de terrain. Dès le 16 mai 1895, il dépose une demande de congé pour entreprendre un voyage en Égypte lors du prochain semestre d'hiver. Grâce à cette démarche, ainsi qu'à une demande de crédit, le projet de Spiegelberg est discuté au plus haut niveau (recteur, curateur) et son nom est désormais associé, dans l'esprit des autorités de l'université, à une expédition qui représentait une aventure moins routinière qu'aujourd'hui. Une fois en Égypte, il fait à Michaelis le récit épistolaire de ses découvertes et lui demande d'intercéder pour obtenir une rallonge de crédits. Le professeur d'archéologie classique, enthousiasmé, s'adresse au curateur, puis, remontant la hiérarchie, au Ministère d'Alsace-Lorraine, en louant les mérites du jeune missionnaire : " Je crois que ces résultats d'une période de travail si courte font un grand honneur au zèle et au flair du Dr. Spiegelberg $"$ "

6. Au retour du voyage, accompli pendant l'hiver 1895/96, l'entourage de Spiegelberg organise la publicité des résultats scientifiques et patrimoniaux de l'opération. Au printemps, le savant ramène en effet d'Égypte des objets authentiques et des photographies, auxquels s'ajoute à l'automne un généreux don de sept caisses d'antiquités que l'archéologue anglais Flinders Petrie a prélevées sur le produit des fouilles qu'il vient de mener, avec son assistant James Quibell, à Nagada, à Ballas (hiver 1894/95) et dans les environs du Ramesseum (hiver 1895/96). Spiegelberg et Petrie avaient lié amitié sur le terrain et se rendaient 
mutuellement service : le savant allemand, excellent philologue, éditait et traduisait les textes découverts par l'archéologue britannique, qui lui avait promis en retour " que lors du partage des résultats des fouilles de Petrie - menées presque chaque année la collection strasbourgeoise devrait elle aussi être prise en considération à l'avenir ${ }^{10}$. Sous la responsabilité de Michaelis, toujours administrateur par intérim de la collection égyptienne, on finance le transport des œuvres de Londres à Strasbourg et on achète le mobilier nécessaire à la bonne conservation $\mathrm{du}$ fonds et à son utilisation comme support d'enseignement pour les cours. Pour faire passer l'addition de ces dépenses imprévues auprès du curateur, l'archéologue très respecté insiste : " des enrichissements de la collection d'une telle envergure ne se représenteront pas si facilement ! ${ }^{11}$ Pour solenniser la générosité du savant anglais et en assurer la plus grande publicité au sein de l'université, Michaelis et Nöldeke proposent, le 29 juin 1897 , de lui attribuer le titre de doctor honoris causa. Le 22 juillet, la Faculté accepte la proposition et fait bientôt établir le diplôme, dont l'honneur rejaillit à la fois sur le bénéficiaire et, bien entendu, sur Spiegelberg, puisque ce rituel académique témoigne de l'excellence des relations scientifiques internationales que son voyage a permis d'établir. Les succès du jeune Privatdozent sont enfin couronnés auprès du public plus large de la métropole alsacienne-lorraine, car un article circonstancié, intitulé Die ägyptologische Sammlung der Universität Straßburg, paraît le 20 décembre 1897 dans le quotidien Straßburger Correspondenz. Le fonds égyptien, écrit le journaliste, " a dernièrement connu un enrichissement tellement significatif qu'à présent la collection égyptologique occupe déjà une position prestigieuse parmi les collections de dimension relativement petite ". La portée médiatique des acquisitions obtenues par Spiegelberg n'est aucunement indifférente à l'administration de l'université, comme en témoigne la coupure de cet article, soigneusement conservée dans le dossier du Kuratorium contenant les " Actes de la Kaiser-WilhelmsUniversität concernant la collection égyptienne ${ }^{12}$.

7. Expérience du terrain, carrure internationale, rayonnement au sein de la société strasbourgeoise les conditions semblent désormais réunies pour engager sous des auspices favorables une demande de recréation de la chaire égyptologique au profit du Dr. Spiegelberg : Nöldeke en fait la proposition le 29 février 1898. Pendant deux mois, la procédure semble dans un premier temps prendre un tour positif. La demande est acceptée par la Faculté, qui transmet la requête au gouverneur d'AlsaceLorraine, compétent pour la nomination des professeurs extraordinaires. La réponse de principe ne doit pas soulever d'objection, puisque la Faculté institue une commission d'experts en vue de proposer une nomination : aux côtés de Windelband (philosophe) et du doyen Henning (germaniste), on y retrouve... Michaelis et Nöldeke. La commission se réunit, le doyen transmet les résultats au Ministère d'Alsace-Lorraine et aux services du gouverneur, qui accorde le 5 mai une audience sur l'« ägyptologische Professur ". Mais ensuite, une inertie durable semble s'installer du côté des autorités politiques. La question de la chaire d'égyptologie est encore discutée lors de deux conseils facultaires et des courriers s'échangent entre le doyen et le curateur, mais rien n'y fait, il faudra attendre encore plus d'un an pour que le "Bureau du gouverneur impérial » se décide enfin à pourvoir le poste.

Que s'est-il passé ? Le principe de la réactivation de la recherche égyptologique parmi les disciplines pilotes de l'université de Strasbourg fut-il remis en question? L'explication la plus probable est à la fois simple et pragmatique : il semblerait qu'au contraire, le gouvernement de l'Alsace-Lorraine, à l'instigation des scientifiques, s'était engagé dans une opération de prestige culturel centrée sur l'égyptologie et sur la papyrologie. Et cette action d'éclat avait supposé un investissement financier exceptionnellement élevé ! En effet, au moment même où la Faculté demandait la réouverture de la chaire d'égyptologie, le recteur de l'université en personne sollicitait un entretien auprès du gouverneur, pour défendre le projet de dépêcher deux savants en Égypte, afin d'y acquérir une précieuse collection de papyrus ${ }^{13}$. Forts de cette introduction, Reitzenstein et Spiegelberg déposent le 21 mars 1898 une demande de crédit pour un montant de 12000 marks, à laquelle le gouverneur du Reichsland promet oralement d'apporter son soutien ${ }^{14}$. Pour éviter de compromettre le projet, c'est dans la plus grande discrétion que le Ministère en étudie alors la faisabilité financière. À la veille de l'assemblée facultaire qui fixera la composition de la commission chargée de proposer un titulaire pour la chaire d'égyptologie, le secrétaire d'Etat von Puttkamer annonce à Reitzenstein l'acceptation de la de- 
mande de crédit : "Son Altesse le Prince gouverneur consent à mettre à (votre) disposition les moyens pour la recherche et pour l'achat de papyrus et de monuments écrits égyptiens et grecs pour un montant de $12000 \mathrm{~m}($ arks $) »^{15}$. Ce budget sera réparti à parts égales entre chacun des deux savants. La somme est énorme - à titre de comparaison, le salaire annuel de Dümichen, qui avait atteint le grade le plus élevé (professeur ordinaire), était de 6 ooo marks en fin de carrière ${ }^{16}$. Bien entendu, Reitzenstein et Spiegelberg se sont engagés à rendre des comptes sur le montant qui leur a été confié, en fixant le délai ultime de leur rapport au $1^{\text {er }}$ mai 1899 . On comprend dès lors pourquoi les autorités reportèrent à plus d'un an la décision de pourvoir la chaire d'égyptologie : le gouverneur attendait tout simplement de voir les brillants résultats promis par les universitaires et, surtout, de lire le bilan scientifique et financier de la mission. 8. Nous avons évoqué dans l'introduction de cet article les bénéfices très remarqués de l'expédition égyptienne menée pendant l'hiver 1898/99. Au retour des savants, il est temps de fonder sur ce succès la conclusion de la stratégie que la Faculté développe depuis plus de cinq ans pour relancer l'enseignement de l'égyptologie. Le 9 mai 1899, Nöldeke - à nouveau lui, ainsi que Henning, qui était doyen lors de la demande de recréation de la chaire, "souhaitent la tenue d'une assemblée facultaire en faveur du professorat en égyptologie ". Ce n'est évidemment pas un hasard si dès le lendemain, Reitzenstein et Spiegelberg signent, avec dix jours de retard, le rapport de mission qu'ils avaient promis au gouverneur un an plus tôt ! Les deux démarches parallèles sont restées intimement liées tout au long de la procédure. Le 16 mai, le doyen Koeppel se fait pressant, car il adresse " une lettre au curateur en faveur de l'attribution dans les plus brefs délais du professorat en égyptologie ". Cette fois l'affaire ne traîne plus, le gouverneur ayant obtenu la démonstration de la fiabilité du candidat proposé par la Faculté et, accessoirement, fait économiser aux finances publiques un an de salaire de professeur en début de carrière. Le curateur pourra bientôt répondre à Koeppel que, le 3 juin 1899, le gouverneur impérial d'Alsace-Lorraine a nommé Spiegelberg professeur extraordinaire, chargé d'un enseignement en égyptologie ${ }^{17}$.

Très logiquement, le nouveau titulaire de la chaire est immédiatement désigné pour administrer la collection égyptienne, ce qui met un terme aux inté- rims exercés successivement par Michaelis et, depuis moins d'un mois, par Dehio (historien de l'art). Mais dans la foulée de la réactivation de la chaire et de la constitution d'une riche collection d'enseignement, Spiegelberg veut marquer le coup en sollicitant la création d'un "Institut d'égyptologie ". En effet, malgré l'existence d'une ligne budgétaire consacrée à l'entretien et au développement du matériel pédagogique permettant d'enseigner l'Antiquité égyptienne, la Faculté de philosophie ne comportait pas à cette époque d'institut d'égyptologie comparable à l'" Institut für Altertumswissenschaft ", à l'" Historisches Institut " ou au " Kunstarchäologisches Institut ». Pour rehausser le prestige local de sa discipline, Spiegelberg souhaite régulariser cette situation en demandant dès le mois de juin " que la collection (égyptienne) soit reconnue comme un établissement de l'université au sens du chapitre VIII du statut de cette dernière et qu'à celui-ci soit attribuée la dénomination 'Institut d'égyptologie' ${ }^{18}$. L'assemblée de la Faculté de philosophie, consultée le 8 juillet 1899 , n'ayant pas soulevé d'objection, la requête est acceptée. Auparavant, la collection - mobilier, livres et moulages ou objets authentiques - était gérée par un professeur désigné par le curateur de l'université ; désormais, cette collection enrichie se transforme en un établissement jouissant, au sein de l'université, d'une certaine autonomie budgétaire, et dont un directeur doit être nommé par le gouverneur d'AlsaceLorraine ${ }^{19}$. La nomination de Spiegelberg dans cette fonction, le 27 juillet 1899, consacre ainsi la naissance officielle de l'Institut d'égyptologie de l'Université de Strasbourg ${ }^{20}$.

Les objets acquis lors des expéditions égyptiennes des hivers 1895/96 et 1898/99 sont conservés dans deux établissements différents : le nouvel Institut d'égyptologie, situé au premier étage du Kollegiengebäude, héberge les objets archéologiques et quelques papyrus et ostraca égyptiens, tandis que les papyrus achetés lors du voyage conjoint de Reitzenstein et Spiegelberg sont logés à la KULB. À leur retour de mission, les deux savants avaient souhaité faire visiter ces trésors papyrologiques au gouverneur, qui avait fourni les moyens financiers de l'opération grâce à son " Dispositionsfonds " personnel (fonds publics dont l'utilisation était laissée à la libre appréciation du gouverneur) : " Les soussignés s'estimeraient heureux s’il leur était accordé, dès que les papyrus auront été déroulés et remontés, 


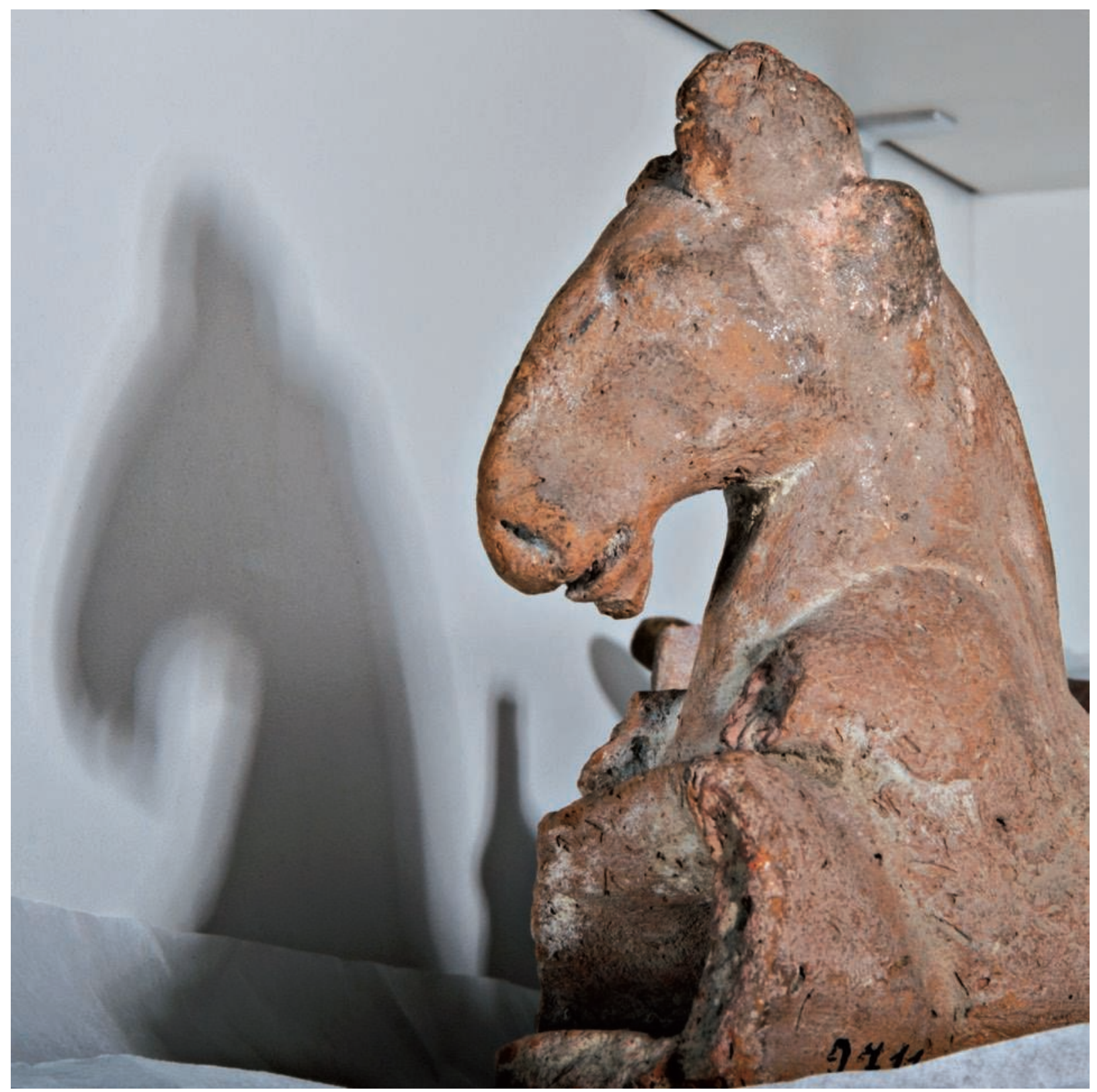

Décor façonné en forme de cheval d'un objet en terre cuite, Égypte. Provenance et circonstances d'acquisition inconnues (coll. UdS) 
de pouvoir faire voir la collection à votre Altesse $"{ }^{21}$. Commence alors, sous l'égide de la KULB, un formidable travail de fourmi pour conditionner et mettre sous verre les innombrables fragments de papyrus achetés grâce au crédit de 12000 marks. D'après les factures conservées dans les archives de la bibliothèque $^{22}$, celle-ci va recevoir à partir du 31 juillet 1899 , pendant plus de sept mois d'affilée, la livraison de centaines de plaques de verre. La mise en place des papyrus, insérés chacun entre deux de ces plaques, fournit un emploi inhabituel au relieur C. B. Simon, à qui seront payées de nombreuses heures de maind'œuvre jusqu'au mois de mars 190o. Lorsque les travaux sont suffisamment avancés, on prend contact avec le bureau du gouverneur et, le 21 décembre 1899, Reitzenstein envoie un billet au directeur de la bibliothèque, Karl August Barack : " Je viens de recevoir une réponse du Prince. Il veut préciser un jour après Noël ${ }^{23}$. La visite officielle, à laquelle seront en outre conviés tous les membres de la Faculté de philosophie ${ }^{24}$, aura finalement lieu le 14 janvier 1900. Cependant Reitzenstein, en mauvaise santé depuis qu'il est revenu d'Égypte frappé d'une " sévère maladie ${ }^{25}$, signale la veille à Barack qu'il est indisposé et craint de ne pouvoir se déplacer : le cas échéant, «le Professeur Spiegelberg devrait avoir l'obligeance de se charger de mon domaine ${ }^{26}$. L'ensemble du fonds papyrologique, égyptien et grec, fut donc peut-être présenté par l'égyptologue seul, lors de la consécration en présence du gouverneur d'Alsace-Lorraine et des collègues admiratifs de la Faculté.

\section{Deux randonnées égyptologiques et papyrologiques en Égypte}

Aux savants et aux amis qui l'ont aidé à poser les premiers jalons de sa carrière, Spiegelberg exprime sa reconnaissance et son admiration en leur dédiant différents travaux au fil des années : on relève notamment, parmi les dédicataires, le maître Dümichen en 1892 ; en 1896, les parents de Spiegelberg; en 1902, les artisans de sa titularisation, Adolf Michaelis et Theodor Nöldeke ; la même année, le sémitisant Julius Euting, nouveau directeur de la KULB ; en 1904, le généreux Flinders Petrie, qui l'avait accompagné dans ses premiers pas sur le terrain ; en 1925, Adolf Erman, rapporteur de sa thèse d'habilitation... En 1909, Spiegelberg dédie en ces termes la première monographie consacrée à la collection de l'Institut d'égyptologie : " dédiée dans un esprit d'amitié à Richard Reitzenstein, en souvenir de la randonnée égyptienne commune il y a dix ans". Pour retracer l'histoire de cette " randonnée " fondatrice de la collection papyrologique de la BNU, on en était jusqu'ici réduit à gloser au départ sur des travaux publiés par Spiegelberg ${ }^{27}$ ou à renvoyer à quelques mots d'introduction d'une étude que Reitzenstein fit paraître en $190{ }^{28}$. Les documents que le Kuratorium de l'université impériale conserva dans les archives liées à la formation et à l'administration des collections égyptiennes jettent désormais un éclairage complémentaire sur cette aventure, en révélant, depuis les coulisses, la façon dont les deux savants concevaient et présentaient leur propre entreprise. Aujourd'hui, les chercheurs consacrent une partie de leur temps à solliciter des crédits auprès des bailleurs de fonds. Les dossiers que nous constituons à cette fin sont appelés à former des gisements documentaires, qui fourniront des sources précieuses pour l'histoire des sciences : les projets accompagnant nos demandes manifestent une rhétorique, une stratégie, des méthodes et des objectifs ; quant aux orientations prises sur le terrain de la réalité et aux efforts de valorisation des résultats scientifiques, ils apparaissent dans les rapports rédigés au terme de nos programmes. Dans un contexte certes moins institutionnalisé qu'aujourd'hui, Reitzenstein et Spiegelberg pratiquaient déjà les règles du genre pour obtenir le soutien financier du gouvernement d'Alsace-Lorraine.

\section{Le cheminement d'un projet de collection}

Le projet de créer une collection de papyrus égyptiens n'est pas né ex nihilo à l'occasion du voyage de l'hiver 1898/99. L'idée s'était fait jour progressivement, en contrepoint des opérations de lancement de la carrière de Spiegelberg, à mesure que le jeune savant découvrait le terrain égyptien. Lors des préparatifs du premier voyage, l'acquisition d'objets authentiques n'est pas envisagée. En sa qualité d’administrateur par intérim de la collection égyptienne, Adolf Michaelis demande le 11 juillet 1895 un crédit exceptionnel de 800 à 1000 marks pour permettre au Privatdozent Spiegelberg - en train d'organiser sa première expédition en Égypte - d'acheter « des pho- 
tographies et des ressources d'enseignement pour le dispositif égyptologique " (doc. 1$)^{29}$.

L'argument essentiel de la requête est économique : le voyage permettra d'acquérir sur place des photographies de monuments égyptiens à un tarif beaucoup plus intéressant que si les commandes devaient se faire depuis Strasbourg. Le projet de constituer un fonds d'images archéologiques est sans doute inspiré par Michaelis lui-même, qui a créé une riche collection de photographies sur le monde classique dans le cadre du Kunstarchäologisches Institut. Pour Spiegelberg, l'objectif est essentiellement pédagogique. Il s'agira, d'une part, "de créer un dispositif utilisable pour des cours et des exercices d'histoire de l'art " (doc. 2). Les lacunes de la documentation accessible pour illustrer ses leçons doivent être comblées car, aux yeux du savant, les publications archéologiques de son temps accordent une place trop réduite aux monuments de l'art profane. En outre, il lui tient à cœur d'accueillir dans sa photothèque, et donc dans ses enseignements à venir, des témoignages artistiques de toutes les époques de l'Antiquité égyptienne, "depuis les temps les plus anciens jusqu'à l'art copte inclus ". D'autre part, la collection de photographies et de ressources pédagogiques devra offrir un "fondement fiable pour des études épigraphiques".

$\mathrm{Au}$-delà de ce programme d'acquisition documentaire très sage et raisonnable, la découverte, quelques mois plus tard, des conditions réelles de l'archéologie égyptienne en cette fin du XIX ${ }^{\mathrm{e}}$ siècle semble avoir agi sur Spiegelberg comme un détonateur. Avec les crédits qui lui ont été promis, il peut faire bien plus qu'acheter des images : le voilà désormais irrésistiblement attiré vers les originaux. Dès son arrivée à Louqsor, le jeune homme de 25 ans, sans expérience antérieure de l'archéologie, se démène en courant littéralement dans toutes les directions à la découverte des vestiges de la rive gauche de Thèbes. Voici comment Michaelis résume les résultats des six premières semaines de l'exploration (doc. 4), dont il vient de prendre connaissance grâce à un courrier envoyé depuis l'Égypte :

1) Copie et estampages de plus de 1000 inscriptions rupestres (graffitis) dont une partie a une importance considérable du point de vue de l'histoire ou de l'histoire de l'art.

2) Découverte des restes de l'implantation de l'ancienne nécropole de Thèbes.
3) Découverte d'un " atelier de choachytes ", dont le Dr. Spiegelberg veut offrir le contenu à notre collection. 4) Découverte d'une tombe d'ibis, qui a été installée à l'époque ptolémaïque dans une tombe de la XVIII ${ }^{e}$ dynastie.

5) Découverte près du fameux Ramesseum du temple d'Amenothes I et de sa mère Ahmes-Nefret-Re, que l'on cherchait en vain depuis longtemps. Cette très importante découverte, qu'il faudra poursuivre davantage, a dû être abandonnée pour l'instant par le Dr. Spiegelberg, faute de temps, de moyens et d'un conseiller en architecture qualifié. Il espère pouvoir gratifier notre collection de quelques-unes des très belles statues du temple.

6) Participation à la découverte du temple de Ramsès, dirigée par Flinders Petrie, dont les bénéfices ont été en grande partie attribués au Dr. Spiegelberg pour étude. Voilà que se transforment les ambitions du programme d'acquisition envisagé au départ ! En menant à titre personnel des fouilles improvisées, le savant découvre des artefacts dont il fera don à la collection égyptienne de l'université. En outre, les possibilités offertes par le marché des antiquités le mènent bien au-delà de ses attentes : avec le budget à sa disposition, il se procure des pièces authentiques (doc. 3). Des photographies sont certes achetées comme prévu - pour un coût de 200 marks - mais un montant de 500 marks est par ailleurs employé à acquérir " une série de beaux bronzes, reliefs en pierre et céramiques ". Les objectifs poursuivis grâce à l'enrichissement de la collection s'élargissent également : " Avec une somme dont le montant évalué n'est pas trop élevé, (Spiegelberg) se fait fort de former une collection qui non seulement satisferait aux objectifs d'enseignement à venir, mais également - en conjonction avec les moulages et les photographies déjà disponibles - pourrait rayonner dans des cercles plus larges " (doc. 3). À la finalité proprement universitaire commence à se superposer, en filigrane, une volonté de diffuser la richesse d'un patrimoine scientifique au-delà des cénacles spécialisés, voire d'inscrire la recherche égyptologique dans le cadre d'une politique de prestige culturel.

\section{La composition d'une équipe}

Le crédit de 12 ooo marks demandé en mars 1898 en vue d'acheter des papyrus fait exploser l'échelle des 
moyens sollicités. Lorsque des scientifiques souhaitent faire financer un programme de recherche, ils doivent soigner la présentation de leur équipe pour convaincre que le projet réunit toutes les conditions nécessaires pour aboutir grâce à leur expérience et aux compétences réunies. Dans le tandem strasbourgeois, " le Dr. Spiegelberg s'est rendu lui-même, voici deux ans, pendant une assez longue période en Égypte et est préparé à une telle entreprise grâce à sa connaissance du pays et de la population et grâce à ses acquisitions pour la collection égyptienne " (doc. 5). Quant à Reitzenstein, ses compétences de philologue classique assureront " la possibilité d'acquérir aussi les papyrus grecs selon un choix également opportun ". À cela s'ajoute, vraisemblablement, le fait que son statut de " professeur ordinaire " garantit à l'expédition un label académique, dont ne peut se prévaloir son jeune collègue Privatdozent - du reste, le ministère signifiera spécifiquement son acceptation au professeur, à charge de transmettre l'heureuse nouvelle au co-équipier non titulaire.

Quelle fut la part respective des deux hommes dans la conception du projet ? Si la demande de crédit est signée conjointement par Reitzenstein et Spiegelberg (doc. 5), le manuscrit est écrit de la plume du premier - façon protocolaire de fiabiliser la requête grâce à l'autorité du professeur ? La configuration inverse s'observe au retour de la mission, en mai 1899, car on reconnaît, dans le rapport (doc. 7), l'écriture de Spiegelberg. Il s'agit désormais, peut-être, de mettre en évidence le candidat à une charge de professeur extraordinaire, à moins, plus prosaïquement, que la sérieuse maladie dont souffre Reitzenstein ne l'ait contraint à céder la plume. Les deux auteurs doivent avoir construit l'argumentation en commun, en fonction de leurs compétences respectives. Mais c'est évidemment la précédente expédition de Spiegelberg, et sa découverte des cours favorables du marché des antiquités, qui inspirèrent l'ambition de créer une prestigieuse collection de papyrus. D'après les publications dont Reitzenstein pouvait s'autoriser au moment de la rédaction du projet, ses thématiques de recherche n'étaient pas spécifiquement orientées vers la papyrologie. Cependant, il accepta volontiers de s'associer à l'entreprise de son cadet, " dans l'espoir de rendre un service à la science allemande ". C'est en effet à Spiegelberg, précise-t-il en 1901, que revient le mérite d'avoir conçu le projet de fonder une collection de papyrus à Strasbourg, afin de trouver un " remplacement pour les trésors qui furent détruits par des boulets allemands en l'année 1870 , à la suite d'un malheureux hasard ${ }^{30}$.

\section{L'objectif politique et patrimonial d'une " bibliothèque de papyrus "}

En appoint à l'intérêt proprement scientifique d'un projet, il est de bonne méthode, pour convaincre audelà du cercle des spécialistes, de le placer sous les auspices de valeurs consensuelles qui transcendent les objectifs immédiats poursuivis par la recherche savante. En l'occurrence, Reitzenstein et Spiegelberg n'hésitent pas à inscrire leur action dans l'esprit et dans la continuité de l'âge d'or des Temps modernes, celui du renouveau de la philologie occidentale autour de la cause commune de l'exhumation et de l'édition de manuscrits grecs : "Cela nous remémore l'époque des Humanistes, où les trésors de bibliothèques helléniques firent revivre les études de l'Occident et où princes et villes, dans un esprit de compétition, prenaient part à l'acquisition de ces trésors" (doc. 5). Le vocabulaire employé, " princes et villes ", n'est pas innocent. On rappellera en effet que le gouverneur de l'Alsace-Lorraine, membre d'une vieille famille princière, est lui-même prince de Hohenlohe-Langenburg. Quant aux deniers publics dont souhaitent disposer les demandeurs, ils sont prélevés sur les impôts des contribuables de la Terre d'empire et destinés à stimuler le rayonnement de sa capitale, Strasbourg, face à d'autres grandes cités culturelles allemandes - Berlin, Leipzig et Munich. En évoquant l'émulation des "princes " et des " cités " de la Renaissance, qui rehaussaient leur prestige culturel en enrichissant les réserves précieuses des bibliothèques, les deux signataires gratifient implicitement d'une comparaison élogieuse les destinataires de la requête. Mais le compliment est simultanément une élégante provocation, puisque pour le mériter, les autorités du Reichsland sont invitées à se montrer dignes de l'évergétisme culturel de leurs augustes prédécesseurs.

Une référence historique en appelant naturellement une autre, l'argumentation du projet plonge ensuite ses racines dans un traumatisme patrimonial profond chez les Alsaciens - et dans un sentiment de culpabilité proportionnel chez les Allemands, celui de la perte des précieux manuscrits conservés 


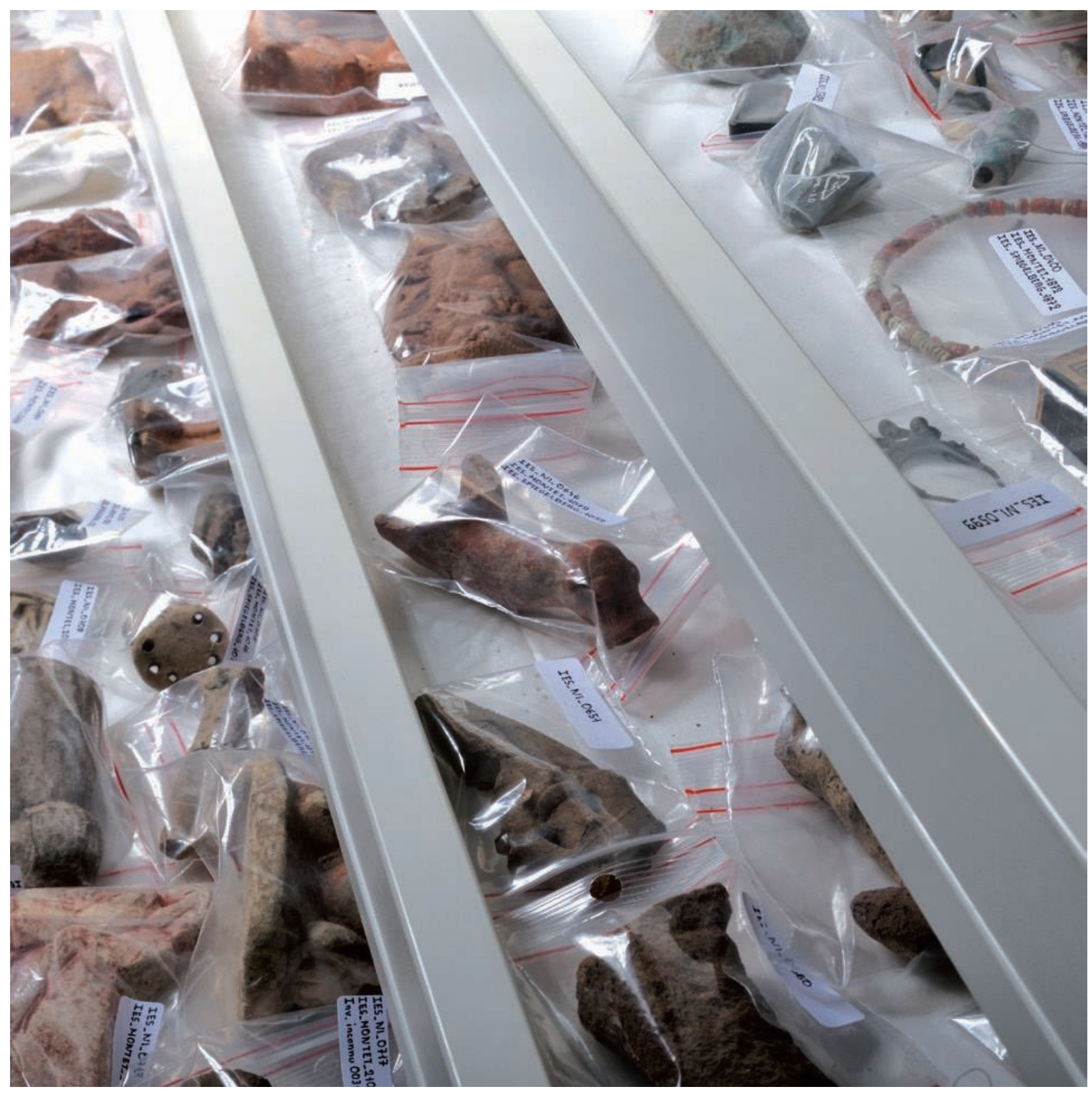

Tiroirs de la collection de l'Institut d'égyptologie de l'Université de Strasbourg, au laboratoire de la MISHA (coll. UdS) 
jadis dans les bibliothèques de l'église des Dominicains, incendiée par les obus des assaillants lors du siège de Strasbourg en $1870{ }^{31}$ : " Dans le Reichsland et à Strasbourg ", insistent les deux universitaires, " nous avons une raison toute particulière de nous rappeler cette époque, parce qu'une riche bibliothèque humaniste a existé ici et n'a disparu que tout récemment. Un remplacement de celle-ci, d'une valeur comparable, s'acquerrait, selon notre conviction, encore sans un sacrifice trop important et de cette façon donnerait à notre bibliothèque splendidement développée - qui du point de vue des imprimés ne le cède qu'à Berlin, Leipzig et Munich - ce qui lui manque encore toujours et qui si souvent est amèrement regretté, y compris par ses directeurs ". Pour offrir à Strasbourg un " Ersatz " des originaux disparus, si l'on suit l'argumentation déployée, il suffirait de s'approvisionner auprès des gisements de manuscrits inédits auxquels la papyrologie, jeune discipline, donne désormais accès.

\section{L'objectif scientifique d'une " bibliothèque de papyrus "}

À leur interlocuteur non spécialiste, les auteurs $\mathrm{du}$ projet commencent par dresser un tableau synthétique des progrès spectaculaires auxquels concourt la papyrologie dans le développement des sciences de l'Antiquité - un exposé fondé sur des publications récentes, qui pourrait introduire un manuel rédigé à la fin du XIX ${ }^{\mathrm{e}}$ siècle :

"Les magnifiques découvertes de papyrus qui ont été faites en Égypte dans les dix dernières années ont apporté un enrichissement insoupçonné à toute une série de sciences. Sans parler de l'archéologie égyptienne, qui leur doit un énorme essor dans tous ses domaines, la philologie classique a accueilli une série d'œuvres écrites inconnues d'une grande importance, des discours du temps de l'apogée d'Athènes, des descriptions de sa constitution et de son histoire, des tableaux de genre inspirés de la vie quotidien- ne de l'époque alexandrine et même, récemment, les chants puissants d'un rival de Pindare à l'époque des guerres médiques ; l'historien a conservé l'administration générale de l'Égypte grecque et romaine à travers la réglementation des impôts du deuxième Ptolémée, le théologien, d'inestimables restes d'anciens Évangiles et d'écrits du premier christianisme, le juriste, des matériaux presque incommensurables pour des études de droit comparé, au traitement desquels des hommes comme Mommsen, Mitteis et d'autres travaillent activement. Même le médecin a pu bénéficier des plus précieux éclaircissements sur l'histoire et le développement de sa science ".

Plus spécifiquement, Reitzenstein et Spiegelberg en viennent à exposer les caractéristiques et les objectifs auxquels, à leurs yeux, devrait répondre la création d'une collection papyrologique au sein de la KULB : «Une bibliothèque de papyrus a, de par sa nature, un double but. Elle devrait, si possible, comprendre d'une part des œuvres littéraires, dont chacune suffirait déjà à classer, aussi de ce point de vue, la bibliothèque qui la possède parmi les premières et les plus importantes d'Allemagne ". Dans la continuité de la tradition humaniste attachée à l'étude des " codices " littéraires, la place d'honneur, sur l'échelle du prestige culturel, est donc réservée aux œuvres des grands auteurs classiques $^{32}$. Mais, les signataires du projet en sont conscients, ils ne peuvent promettre qu'ils auront à coup sûr l'occasion d'acquérir une pièce maîtresse d'une telle valeur dans le courant du prochain hiver. Néanmoins, une péripétie - dont les archives du Kuratorium nous livrent le "scoop " à plus d'un siècle de distance - leur donne quelque espoir : "Le Dr. Reinhardt (leur contact au Caire) aurait pu acquérir pour nous l'inestimable papyrus de Bacchylide pour 6000 marks ". La découverte de ce texte dévoilant un pan entier d'une œuvre perdue, finalement acheté pour le compte du British Museum et édité un an avant la rédaction du projet, avait immédiatement bénéficié d'un retentissement exceptionnel 
bien au-delà des milieux de la papyrologie. Mais qu'à cela ne tienne, l'occasion manquée est révélatrice de la nécessité urgente qu'il y aurait de confier aux deux Strasbourgeois des fonds suffisamment importants pour pouvoir réagir efficacement aux offres du marché : "Il s'agit pour de tels cas, où il est indispensable de sauter rapidement sur l'occasion, de disposer d'un crédit pour une assez grosse somme ". Le second objectif que devrait poursuivre la future collection strasbourgeoise serait de constituer un corpus documentaire polymorphe : " D'autre part, une telle bibliothèque de papyrus devrait aussi comprendre la multitude bigarrée de plus petites pièces écrites, documents privés et publics, comptes rendus, courriers, notes ou exercices scolaires, qui nous présentent la situation sociale, économique, culturelle et religieuse du plus lointain passé au travers d'une vision vivante et immédiate que l'on ne soupçonnerait pas et qui grâce à cela raniment la science ". En illustration de cette catégorie de documents, les collections déjà fameuses de Vienne et de Berlin sont alors citées en exemple pour la valeur qu'elles ont fondée sur des pièces manifestement présentées comme secondaires : " La collection de son Altesse impériale et royale l'archiduc Rainer, comme celle du musée de Berlin, ont eu un énorme impact et sont d'une grande importance, sans posséder une seule de ces pièces de premier rang ". L'ombre d'un défi point-elle derrière cet éloge à double tranchant - nous sommes avant l'expédition, Strasbourg peut encore espérer se distinguer face aux capitales de la papyrologie germanique grâce à une acquisition sensationnelle ? Les candidats au voyage concluent ensuite leur programme prévisionnel par une réflexion véritablement prophétique pour le développement ultérieur du fonds strasbourgeois : "Sans compter que, dans cette diversité de petites pièces se trouvent toujours aussi des fragments plus courts d'œuvres littéraires, qui ont déjà permis assez souvent des conclusions d'une grande portée sur des genres littéraires perdus ». Le lecteur d'aujourd'hui ne peut que sourire en songeant à L'Empédocle de Strasbourg, dont les fragments à première vue modestes, achetés en 1904 par l'intermédiaire du Deutsches Papyruskartell, ne furent identifiés qu'en 1994 comme le seul témoin en tradition directe d'une œuvre présocratique majeure ${ }^{33}$.

\section{Les conditions d'acquisition sur le terrain : le produit des fouilles}

Les archives de la Kaiser-Wilhelms-Universität jettent quelques lueurs sur la façon dont Wilhelm Spiegelberg appréhendait le travail de terrain et sur les circonstances dans lesquelles il s'y approvisionnait au profit des fonds égyptiens de Strasbourg. Nous l'avons déjà vu, à l'occasion de son premier voyage, mener des fouilles personnelles improvisées (doc. 3 et 4). Logeant dans une maison du hameau de Dra Abou el-Naga, dont l'habitat recouvre la nécropole thébaine sur les premières pentes de la montagne Libyque, il rayonne en réalité au départ de ce camp de base à la recherche d'inscriptions rupestres. Comme cela arrive à tout archéologue sillonnant la surface d'un site funéraire en Égypte, sa prospection pédestre le mène vraisemblablement à observer, traînant à même le sol, des fragments d'objets provenant du mobilier funéraire de tombes pillées. C'est peut-être dans de telles circonstances qu'à deux pas de Dra Abou el-Naga, sur le chemin menant à la vallée des Rois, il pousse plus loin son examen en vidant un puits aménagé dans un ouadi : l'Inventaire de l'Institut d'égyptologie comporte une liste de 41 artefacts sous le titre "Objets issus d'un puits (magasin d'un embaumeur ?) dans le désert thébain (Wadi 'En) ouvert par le Dr. Spiegelberg en décembre 1895 ". Encore plus près du village, il entre, quelques décennies après Champollion et Lepsius, dans la tombe de Héry (Tombe Thébaine 12) pour y copier des graffitis. Mais l'Inventaire de l'Institut confirme une fois encore que l'égyptologue ne se contente pas de faire de l'épigraphie, puisque $14 \mathrm{nu}$ méros y répertorient les "Trouvailles de la tombe de Hray ouverte en janvier 1896 ". En bonne méthode, Spiegelberg s'en remet aussi aux compétences d'un paysan du village, pour déceler les vestiges archéologiques affleurant un peu partout sur le sol - cet " excellent Idris Awad, probablement le meilleur connaisseur de la nécropole thébaine parmi ses habitants actuels "34. Les deux hommes prospectent les terres en contrebas de la montagne, parce que Spiegelberg soupçonne que s'y cachent les ruines du temple funéraire d'Amenhotep ${ }^{\text {er }}$, dont l'emplacement est encore inconnu. Et en effet, à la limite des terres cultivées, son guide repère bientôt une première pierre. Les plus gros blocs, ceux qui nécessitent pour être retournés l'usage des outils prêtés 
par Flinders Petrie - qui fouille au même moment le Ramesseum - Spiegelberg les laisse bien entendu sur place. Mais une dizaine d'autres fragments de reliefs et de statues, de petites dimensions, sont ramenés à Strasbourg. Ces pièces seront publiées beaucoup plus tard par Philippe Derchain, directeur de l'Institut de 1964 à 1968, qui évoque non sans une pointe de dédain la découverte du savant allemand : " Les misérables ruines du temple d'Aménophis Ir et d'Ahmes Nefertari que l'on peut encore voir à proximité du point d'eau où se ravitaillent les femmes de Dra Abou el-Naga n'ont certes pas de quoi attirer le visiteur $" 35$.

Après cette expérience scientifique en partie improvisée, qui lui a fait prendre conscience du potentiel offert par le périmètre prospecté, Spiegelberg planifie pour l'hiver 1898/99 une campagne plus systématique avec la collaboration d'un collègue anglais, Percy Newberry, dont il a fait la connaissance lors de son premier séjour thébain. Cette fois, les fouilles sont menées pour le compte d'un mécène, le marquis de Northampton. Grâce à ce financement privé, des travaux intensifs, avec un nombre important d'ouvriers, sont dirigés du 7 novembre au 27 janvier dans plusieurs secteurs de Dra Abou el-Naga. À nouveau, les recherches se déploient au départ du village, où la maison d'Idris Awad sert régulièrement de point de repère topographique dans le Journal des découvertes de Spiegelberg. Ce manuscrit rédigé au jour le jour par l'égyptologue strasbourgeois, aujourd'hui conservé au Griffith Institute (Oxford), laisse entrevoir l'empirisme qui prévaut dans la mise en place progressive des méthodes de fouilles. Génial déchiffreur de toutes les écritures de l'égyptien ancien, Spiegelberg l'autodidacte est encore en plein apprentissage en ce qui concerne l'archéologie de terrain. D'une façon touchante, il écrit à la suite d'une discussion avec un architecte britannique expérimenté, de trente ans son aîné : " 15 novembre (...) Visite de M. Somers Clarke, qui nous a donné de bons une série d'excellents tuyaux. Il faut surtout que les matériaux de construction soient aussi notés sur le plan ". Et sans doute, les conseils portent aussi sur les techniques d'enregistrement des trouvailles, car c'est précisément à partir de cette date que l'organisation du Journal des découvertes est mise en place... Un autre jour - le 3 décembre - c'est Howard Carter, futur découvreur de la tombe de Toutankhamon, qui vient donner son interprétation sur un phénomène stratigraphique un peu complexe. D'une façon générale, Spiegelberg est soucieux de tenir compte de l'ensemble du mobilier mis au jour et se montre notamment sensible à la description des vases en terre cuite, dont la valeur de "fossiles directeurs " - permettant de dater les contextes fouillés - deviendra fondamentale dans l'archéologie moderne. Au terme de la campagne, le marquis de Northampton, financeur privé de l'opération, autorise Spiegelberg à offrir une partie du produit des fouilles à la collection de l'université de Strasbourg : les " Objets issus des fouilles de Drah-Abu'l Negga et Gurnah, hiver 1898/99 " occupent en effet plusieurs pages de l'Inventaire de l'Institut. Pour permettre cette importante donation, le Kuratorium devra seulement prendre en charge les dépenses d'emballage et de transport, pour un montant prévisionnel de 1000 marks (doc. 6). Parallèlement à la fouille de la nécropole thébaine, Spiegelberg ne perd pas de vue la mission de chasse aux papyrus dont il a été chargé avec son collègue Reitzenstein. Les deux hommes sont censés employer à des achats sur le marché la somme qui leur est confiée, mais leur rapport révèlera qu'ils ont failli entreprendre des fouilles improvisées pour le compte du gouvernement du Reichsland. " À deux reprises ", écrivent-ils au gouverneur d'Alsace-Lorraine, " des fouilles mineures ont été envisagées mais ont été abandonnées pour des raisons déterminées. À une occasion, les indications prometteuses de savants qui croyaient avoir découvert un pendant de la bibliothèque d'Herculanum se sont révélées fausses in situ ". On imagine l'excitation des deux savants, qui ont cru un temps pouvoir mettre la main sur une découverte exceptionnelle - un pendant nilotique des volumes de textes littéraires conservés sous les cendres du Vésuve, dont l'exhumation au milieu du XVIII ${ }^{e}$ siècle avait consacré la naissance de l'intérêt scientifique pour les papyrus grecs ${ }^{36}$. Ils se rendent sur place, grattent le sol qu'on suppose cendreux, et puis : rien. Un coup du sort leur fait manquer une seconde occasion : " Un lieu de découverte indubitablement majeur, que pistait un Arabe œuvrant déjà depuis longtemps à nos projets, est malheureusement resté inexploitable pour nous à cause de la mort soudaine de ce dernier. Par conséquent seule demeura la possibilité de l'acquisition sur le marché " (doc. 7). 


\section{Le chercheur de papyrus a quelque affinité avec un pêcheur qui ne jetterait ses filets qu'avec discrétion pour ne pas attirer l'attention sur les eaux les plus poissonneuses}

\section{Les conditions d'acquisition sur le terrain : le marché des antiquités}

Comme ces récits le laissent entrevoir, à la fin du $\mathrm{XIX}^{\mathrm{e}}$ siècle, les égyptologues travaillent dans des conditions éloignées des méthodes de prospection actuelles et leurs relations au patrimoine archéologique égyptien ne sont pas encore encadrées par les normes déontologiques et réglementaires qui s'imposeront progressivement par la suite. Il serait cependant anachronique de juger avec les valeurs d'aujourd'hui les pratiques d'un autre temps, où les exportations d'antiquités étaient officiellement taxées par les autorités et où les protagonistes trouvaient leur compte en toute légalité. Le chercheur de papyrus, à cette époque, a quelque affinité avec un pêcheur qui ne jetterait ses filets qu'avec discrétion pour ne pas attirer l'attention sur les eaux les plus poissonneuses. Le marché est régi par les lois de l'offre et de la demande, il faut à la fois disposer de fonds élevés pour pouvoir l'emporter sur les concurrents et éviter de faire monter les prix par une imprudente ostentation de ses moyens. En 1898, Reitzenstein et Spiegelberg suivent scrupuleusement ces principes, lorsqu'ils mettent en route la procédure de demande de crédits : " Comme la première condition du succès est qu'une telle mission d'achat demeure totalement secrète, le mieux, d'un point de vue pratique, serait peut-être de faire verser cette somme par un truchement quelconque à une maison bancaire assez importante comme par exemple le "Crédit lyonnais " au Caire et ce à raison de la moitié (de la somme) pour chacun des deux soussignés " (doc. 5). Pour souligner la nécessaire confidentialité de la démarche, Reitzenstein appose lui-même la mention " Geheim " (secret) à côté de l'adresse de sa requête épistolaire. La demande de discrétion est prise au sérieux. En marge du paragraphe en question, un responsable de l'administration ajoute une accolade pour en signaler l'importance, et le bureau du gouverneur ordonne au Ministère d'Alsace-Lorraine d'étudier la demande des requérants dans le plus grand secret : " Monsieur le gouverneur enjoint de traiter l'affaire de façon tout à fait confidentielle " ${ }^{37}$. Le Staatssekretär von Puttkamer s'exécute, car sur le formulaire d'accompagnement du dossier transmis au département des finances sont ajoutés deux mots au crayon rouge : "Secret " (souligné une fois) et "Urgent" (souligné deux fois) ${ }^{38}$.

Ces précautions de conspirateurs sont dictées par l'atmosphère de vive compétition internationale régnant sur le marché des antiquités contemporain. " Car les meilleurs connaisseurs anglais et allemands de la situation ", s'alarment Reitzenstein et Spiegelberg, "sont persuadés que l'étonnante foison de monuments sur le sol égyptien ne peut plus persister que pendant peu d'années, car les chercheurs anglais et malheureusement désormais aussi les entrepreneurs américains s'approprient toujours plus les trésors de papyrus disponibles et le commerce" (doc. 5). Comme on le voit, les savants allemands situent leurs véritables concurrents internationaux dans le monde anglo-saxon - représentant à leurs yeux un modèle d'efficacité dont les succès récents devraient inciter l'université de Strasbourg à se lancer d'urgence dans la compétition. Les dossiers papyrologiques retenus dans le projet pour illustrer "les magnifiques découvertes de papyrus qui ont été faites en Égypte dans les dix dernières années " (doc. 5), que nous avons énumérés plus haut, sont révélateurs de la supériorité concédée aux institutions britanniques. En 1888, le British Museum avait en effet envoyé l'égyptologue et orientaliste Wallis Budge en Égypte, afin d'enrichir ses collections en se fournissant sur le marché local des antiquités. Le musée londonien était ainsi entré en possession d'une exceptionnelle moisson de textes littéraires disparus, que le philologue Frederic Kenyon avait commencé à déchiffrer et à éditer en $1890^{39}$. Le lot livra les plaidoiries et manifestes politiques de grands orateurs athéniens, Isocrate, Démosthène, Hypéride - ce sont les " discours du temps de l'apogée d'Athènes " 
évoqués par Reitzenstein et Spiegelberg. De même, on identifie aisément sous leur plume " les descriptions de sa constitution et de son histoire " comme l'Athènaiôn politeia, dont la publication par Kenyon en 1891 révolutionna la connaissance des institutions athéniennes. La même année, le chercheur anglais enchaîna sur l'édition des Mimes d'Hérondas, que les deux Strasbourgeois définissent comme " des tableaux de genre inspirés de la vie quotidienne de l'époque alexandrine ». En 1893/94 et en 1894/95, Flinders Petrie et Bernard Grenfell acquirent, pour le compte de la Bodleian Library (Oxford), le papyrus des Revenue Laws, publié par Grenfell en 1896 ; pour reprendre les termes de la requête au gouverneur, ce document fiscal permet à l'historien d'étudier « l'administration générale de l'Égypte grecque et romaine à travers la réglementation des impôts du deuxième Ptolémée ». Quant au célèbre papyrus de Bacchylide contenant « les chants puissants d'un rival de Pindare à l'époque des guerres médiques " - papyrus qui aurait pu être acheté par Strasbourg - il aboutit en 1896 dans les collections du British Museum, pour être publié l'année suivante à nouveau par Kenyon. $\mathrm{Au}$ travers des références implicites auxquelles il renvoie, le plaidoyer de Reitzenstein et Spiegelberg sur les apports de la papyrologie aux sciences de l'Antiquité est donc en soi un témoignage historique sur l'extraordinaire retentissement dont avaient bénéficié les acquisitions et les publications d'une poignée de savants anglais - en particulier pour le compte du British Museum - dans la dernière décennie du XIX ${ }^{\mathrm{e}}$ siècle.

Dans ce contexte de vive émulation et sous la pression d'un marché en voie de saturation, la mission strasbourgeoise doit trouver un angle d'attaque pour s'introduire rapidement sur les plaques tournantes du commerce. Elle peut compter à cette fin sur un atout providentiel : un ancien étudiant de l'université occupe en effet la fonction de premier drogman auprès du Consulat allemand au Caire, poste qui lui a permis d'acquérir une expérience inégalée du marché des antiquités : "Strasbourg serait justement déjà en mesure de pouvoir entrer dans la compétition avec un certain espoir de succès. Un élève du professeur Nöldeke et docteur de notre université, Reinhardt, est employé comme premier drogman auprès du Consulat au Caire et est devenu, au travers de (son expérience) de collection et de recherche longue de quinze ans, le meilleur connaisseur de cette situation et de ce commerce. Il nous a assuré avec beaucoup d'ardeur son soutien énergique pour une collection de papyrus strasbourgeoise " (doc.5). Et c'est en effet grâce à cette aide consulaire que, selon le rapport de mission de Reitzenstein et Spiegelberg, ceux-ci " ont tenu sous une surveillance constante le commerce des antiquités égyptien sur ses deux marchés principaux du Caire et de Louqsor de la fin octobre à la mi-mars et, en outre, ont ratissé lors d'excursions longues et courtes les lieux de découvertes dans les environs du Caire, le Fayoum, Achmîm, Esna, Kena, Roda et Achmounein " (doc.7). Par l'entremise du vice-consul C. Reinhardt, les deux savants se sont ainsi initiés aux arcanes du marché des antiquités, dont les lieux de vente se répartissent en deux niveaux hiérarchiques : les sites archéologiques proprement dits, connus pour leurs gisements de papyrus et d'ostraca, et les plaques tournantes principales du commerce - Le Caire et Louqsor - où les trouvailles sont ensuite acheminées en direction des acheteurs potentiels. Forts de cette expérience, les Strasbourgeois ont désormais établi leurs propres contacts avec les vendeurs, dont ils comptent bien tirer parti lors de leurs prochaines expéditions : " Les relations nouées cet hiver avec des marchands arabes et grecs, et la perspective presque certaine que l'un des soussignés voyage de nouveau en Égypte de temps en temps laissent espérer que se présente encore souvent une occasion d'achats opportuns et en particulier que la partie littéraire de la collection soit encore accrue " (doc. 7).

\section{Les retombées scientifiques et patrimoniales}

Il est temps de faire le point, avec les acteurs de ces aventures, sur les fruits qu'ils retirèrent des expéditions menées lors des hivers 1895/96 et 1898/99. Nous ne reviendrons que brièvement sur les libéralités dont Flinders Petrie et le marquis de Northampton firent bénéficier l'Institut d'égyptologie de l'université de Strasbourg. Leurs dons très importants ont constitué le noyau dur sur lequel se sont greffées les acquisitions ultérieures. Dans les années suivantes, en effet, l'Inventaire de l'Institut répertoriera encore les noms de nombreux donateurs : des collègues et des savants connus ou des mécènes occasionnels de l'entourage de Spiegelberg - voire encore des associations comme le Club vosgien et la Deutsche Orient- 


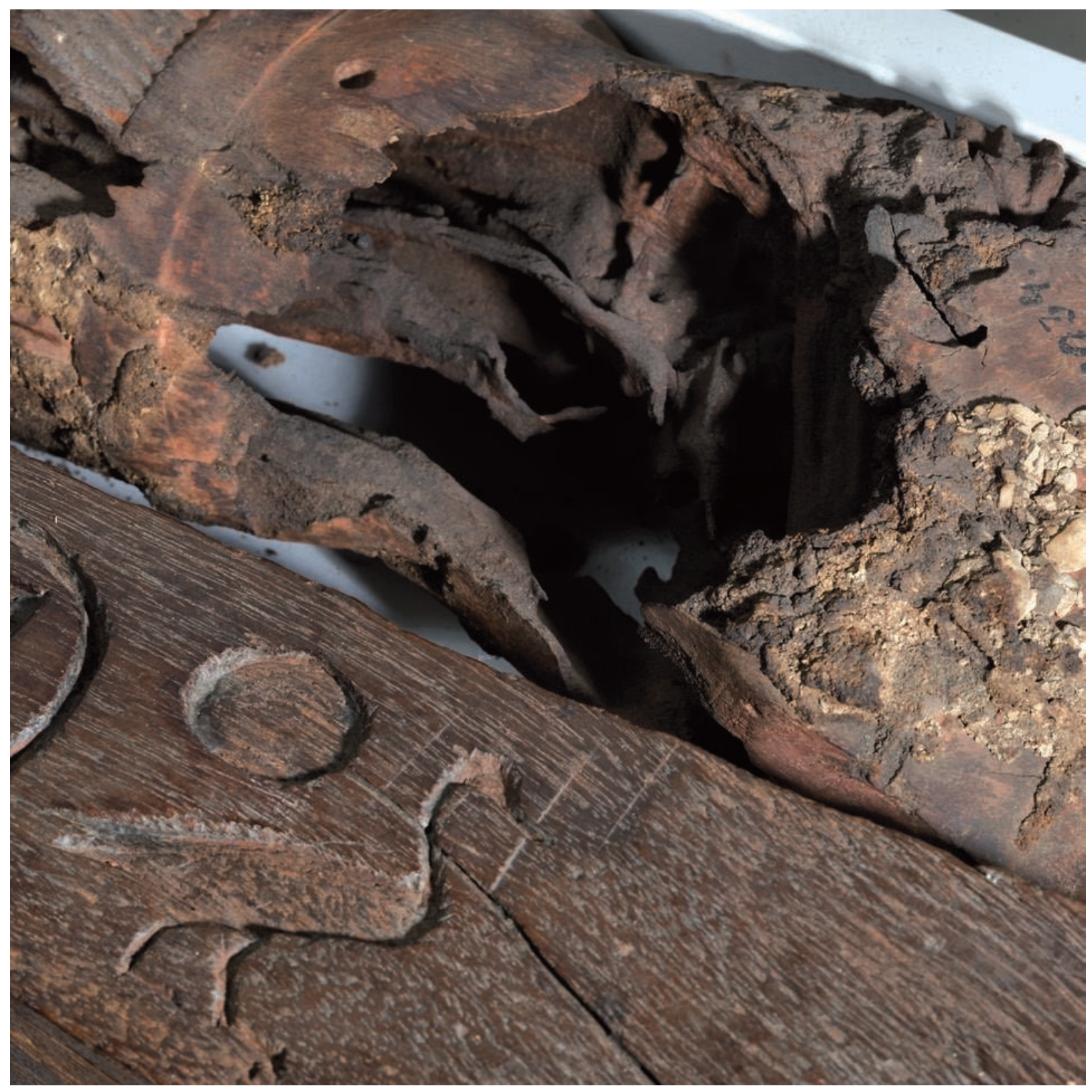

Les ravages inégalitaires du temps : queue d'aronde (expédition de 1895/96, marché des antiquités) et statue de pharaon en bois (expédition de 1898/99, fouille Northampton, Newberry, Spiegelberg) (coll. UdS) 
Gesellschaft ou des musées allemands et étrangers (Berlin, Bonn, Liverpool et Bruxelles).

Pour Spiegelberg, les deux voyages sont l'occasion de s'immerger dans le milieu cosmopolite de l'égyptologie de terrain en Égypte. Au moment de lancer sa carrière et de fonder sa crédibilité locale sur un rayonnement scientifique international, les liens personnels qu'il tisse ainsi durablement, en particulier avec des savants britanniques, ont probablement autant de valeur que les bénéfices proprement patrimoniaux et documentaires de l'opération. À ce propos, il terminera seulement en 1919, quelques mois après la prise de Strasbourg par les troupes françaises, la publication des graffitis copiés en 1895/96. Lorsqu'il signe les avant-propos, le 7 janvier 1919 - à cette date, il s'est déjà fait notifier son expulsion vers l'Allemagne - c'est vers l'âge d'or de son premier voyage en Égypte que le professeur strasbourgeois tourne ses pensées : " Aujourd'hui, au moment d'achever ce travail, mes pensées cheminent en arrière vers le temps où je dépistais les inscriptions avec la joyeuse hardiesse de la jeunesse. C'était encore la belle époque de l'harmonie scientifique, vers laquelle je jette aujourd'hui un regard rétrospectif comme vers un paradis perdu. De combien d'aide ce travail n'a-t-il pas bénéficié de la part des confrères anglais! " ${ }^{40}$. Ensuite, Spiegelberg se remémore avec reconnaissance Percy Newberry, qui l'avait invité à loger dans sa maison de Dra Abou elNaga, Flinders Petrie, pour ses conseils et son aide et Idris Awad, dont le flair avait permis la découverte de si nombreuses inscriptions. "Avec une gratitude toute particulière ", écrit-il encore, " je repense au cercle des égyptologues qui se rassemblait à cette époque autour de Flinders Petrie au Ramesseum, dans lequel chaque jour après l'achèvement de mon travail sur les graffitis je vécus des heures inoubliables ". L'intensité des rapports sociaux que les savants entretenaient sur le terrain, parallèlement à la direction des fouilles, transparaît dans le journal que Newberry écrivit pendant la durée des travaux menés en collaboration avec Spiegelberg ${ }^{41}$. Le 3 janvier 1899, par exemple, Newberry, Reitzenstein et Spiegelberg se rendent au musée du Caire où ils rencontrent une belle brochette d'égyptologues : Erman, Brugsch, Borchardt, von Bissing, Quibell et Chassinat ! Deux jours plus tard, de retour à Dra Abou elNaga, Newberry, Spiegelberg et leur raïs négocient avec le Français Daressy, représentant le Départe- ment des Antiquités, pour délimiter leur concession de fouille. Les deux archéologues reçoivent régulièrement des visites de courtoisie " to lunch ", "for tea " ou " to dinner ". Lisons ainsi la notice du 2 février : " Schweinfurth (et) Northampton sont venus l'après-midi et après le dîner Spiegie et moi sommes allés chez Carter (et) avons joué au whist. Lettre de Grenfell. Arrivée de Kahun Papyri III pour Spiegie ". Ce sont quelques bribes d'une journée au paradis perdu, la réception d'une lettre postée par un génie de la papyrologie britannique, l'arrivée d'un ouvrage scientifique offert à "Spiegie " par un collègue, la visite d'un célèbre explorateur allemand et du diplomate anglais finançant la fouille, une partie de cartes entre trois jeunes prodiges de l'égyptologie tandis que la nuit tombe sur la nécropole thébaine...

Parallèlement aux acquis proprement scientifiques, la " fouille en Égypte ", dans les conditions du XIX siècle, conférait à son auteur une sorte de surplus d'autorité intellectuelle dans le monde des égyptologues, à l'image du " hadj " revenu d'un pèlerinage à La Mecque. Cette plus-value du curriculum vitae de Spiegelberg au retour du terrain est clairement mise en évidence sous la plume de Jean Capart, conservateur adjoint aux Musées royaux des arts décoratifs et industriels de Bruxelles. Dans un courrier interne adressé en janvier 1903 au conservateur en chef Eugène van Overloop, son supérieur, le savant belge valorise ses propres travaux grâce à une expertise établie par son collègue strasbourgeois - ce faisant, il est dans son intérêt d'attester les compétences de son éminent correspondant : "Le professeur Spiegelberg de Strasbourg, un des maîtres de l'école allemande, a visité nos collections à mon retour d'Égypte et vous a envoyé à ce sujet un court rapport. Je tiens à faire remarquer que la compétence du professeur Spiegelberg en ces matières ne peut être contestée. Élève du célèbre professeur Dümichen, $M^{\mathrm{r}}$ Spiegelberg fit des études également à Paris où il suivit naguère les cours de M. Maspero ; il connaît par des séjours prolongés les diverses collections d'Europe et a fait de nombreux voyages en Égypte où il fit encore il y a quatre années |il exécuta/ des fouilles pour le marquis de Northampthon (...) $"{ }^{42}$. L'aventure thébaine de 1898/99, et l'expérience archéologique de terrain qu'elle suppose, occupe désormais une place de choix sur la carte de visite scientifique du " professeur Spiegelberg de Strasbourg ".

Puisque nous en sommes venus à considérer la rhé- 
torique de mise en valeur des travaux scientifiques, laissons à Reitzenstein et Spiegelberg le soin de présenter eux-mêmes les résultats de leur mission de chasse aux papyrus :

"Bien que nous ayons surtout dirigé notre attention sur de grands papyrus littéraires, du même genre que ceux qui étaient apparus il y a plusieurs années - mais il est vrai, de façon tout à fait momentanée sur le marché des antiquités, nos recherches de telles pièces rares sont cependant restées sans succès " (doc. 7).

Autant le dire d'entrée, les rêves de manuscrits littéraires prestigieux, qui à eux seuls hisseraient la bibliothèque strasbourgeoise au premier rang des institutions culturelles de l'Allemagne, ne se sont pas réalisés! Pour l'acquisition de colonnes complètes d'orateurs athéniens, de poètes alexandrins, de rivaux de Pindare - un argument central du projet de compensation des manuscrits perdus en 1870 - il ne reste plus qu'à attendre une prochaine campagne... Mais qu'à cela ne tienne.

" D'autant plus fructueux furent nos efforts en vue de trésors de papyrus d'autre nature. Car à cet égard s'est présenté un matériel étonnamment riche, composé de fragments d'œuvres littéraires petits mais extrêmement importants pour la science, d'une valeur si remarquable que les soussignés se sont crus autorisés, et même forcés d'utiliser la plus grande partie de la somme mise à leur disposition. Ainsi furent acquises environ 2000 pièces grandes et petites, parmi lesquelles se dégagent déjà maintenant les pièces plus importantes suivantes. 1) dans le domaine égyptologique, une assez grosse collection d'ostraca (documents sur des tessons) hiératiques de l'époque ramesside (vers 1200 av. J.-C.), une série de documents sur papyrus en démotique ancien du huitième siècle avant le Christ, tels qu'il s'en présente peu même dans les plus grandes collections égyptiennes. De l'époque plus tardive doivent être mentionnés une assez grande pièce littéraire avec un hymne à Osiris et surtout des documents juridiques de l'époque ptolémaïque d'une étendue tout à fait inhabituelle et dans un état de conservation excellent, et pour finir une collection d'ostraca et de petites tablettes en bois démotiques. Très importants paraissent en outre les papyrus coptes, parmi lesquels une série de précieuses pièces littéraires à contenu théologique a déjà été identifiée grâce à un examen superficiel. Également les lettres, les textes magi- ques et les documents juridiques, en grande partie conservés dans un excellent état, semblent promettre de riches bénéfices. Doit encore être mentionné un papyrus araméen, qui selon toute vraisemblance est conservé de façon complète et ainsi prend la première place parmi les documents de ce genre qui ont été identifiés jusqu'à présent, de même que quelques documents pahlavi (de l'époque sassanide), qui doivent également être signalés comme tout particulièrement précieux. Moins remarquables apparaissent jusqu'ici les monuments écrits arabes et hébreux. 2) Dans le domaine du grec doivent surtout être mentionnés une pièce d'une étendue d'environ sept colonnes, quoique fortement abîmée, d'un auteur semble-t-il inconnu, d'assez longues pièces d'un poème épique tardif nouveau pour nous, un petit fragment d'un poème perdu d'Hésiode, plusieurs fragments de comédies grecques, des pièces d'une nouvelle anthologie et de traités grammaticaux et médicaux de même que de plus petits fragments de différents autres auteurs. Parmi les nombreux documents se distinguent particulièrement huit pièces d'archive de l'époque ptolémaïque, complètement conservées et pour certaines de grandes dimensions, un assez long document testamentaire de l'époque romaine, trois grands rouleaux, toutefois assez abîmés, avec l'allivrement d'une petite ville, enfin un nombre de fragments de lettres impériales. D'une façon annexe, une assez longue lettre latine complètement conservée peut également être mentionnée comme une rareté toute particulière et comme intéressante à plus d'un point de vue ".

Cette première description provisoire de la collection révèle un ensemble documentaire et littéraire d'une très grande valeur scientifique, qui promet des recherches fructueuses pour des décennies de papyrologues - bien au-delà du changement de souveraineté sur l'Alsace, qui menace sans qu'ils le sachent les savants allemands. Dans cette liste à la Prévert, le spécialiste reconnaît des lots qui ne seront publiés que beaucoup plus tard, le plus souvent sous le régime français, grand bénéficiaire du butin papyrologique ramené grâce aux crédits alloués par le gouvernement du Reichsland. Encore dernièrement, le 12 mai 2010, a été soutenue à la Maison interuniversitaire des sciences de l'homme-Alsace une thèse intitulée Un dossier fiscal hermopolitain d'époque romaine conservé à la Bibliothèque nationale et universitaire de Strasbourg ${ }^{43}$. Il est vraisemblable que ce bel ensemble de 
fragments provenant de trois volumina, qui ont demandé à leur déchiffreur des trésors de science et de patience à cause de la grande détérioration du papyrus, puisse être identifié avec le document fiscal que les deux signataires du rapport définissaient comme " trois grands rouleaux, toutefois assez abîmés, avec l'allivrement d'une petite ville ".

\section{Les mystérieuses malles du steamer Silesia : retombées patrimoniales et matrimoniales?}

Le printemps 1899, au retour d'une mission prestigieuse menée avec succès, est pour Spiegelberg la saison de toutes les promotions sociales et professionnelles. Mais sur le plan existentiel, sa désignation comme professeur extraordinaire, puis comme directeur d'un Institut d'égyptologie spécialement créé autour de la collection d'antiques acquise par ses soins, ne sont vraisemblablement pas les plus vitales des transformations de son statut. Dès le 4 avril, en effet, il épouse Elisabeth, fille du célèbre médecin Friedrich Daniel von Recklinghausen, titulaire de la chaire de pathologie et ancien recteur de la Kaiser-Wilhelms-Universität de Strasbourg. Ce mariage endogamique contribuera vraisemblablement à renforcer et à élargir le réseau social du jeune savant au sein de l'université impériale, au-delà de la Faculté de philosophie. Un détail insolite, caché au fin fond d'un dossier de comptabilité, pourrait du moins le suggérer. Le 9 mai, la société de transport " Grewe \& Co ", sur les instructions de Spiegelberg, adresse au secrétaire du Kuratorium une facture de 204,75 marks pour l'acheminement de dix malles depuis Le Caire jusqu'à Brême. Les caisses, embarquées sur le steamer Silesia, que l'on attend dans les prochains jours, sont énumérées comme suit : " 5 malles d'antiquités pour la collection égyptologique ", " 3 malles de même contenu pour l'Institut d'anatomie ", " 2 malles d'équipements etc. pour Monsieur le Dr. Spiegelberg " ${ }^{44}$. Sans surprise, l'université remboursera seulement le transport des huit caisses qui lui sont directement destinées - Reitzenstein et Spiegelberg s'étaient engagés à prendre en charge eux-mêmes leurs propres frais de voyage. Mais pourquoi adresser trois cantines à l'Institut d'anatomie, quelle sorte d'" antiquités " égyptiennes peuvent-elles bien contenir ? Une autre pièce comptable du même dossier contribue à résoudre cette énigme : il s'agit d'un tableau récapitulant les dépenses effectuées au bénéfice de la collection égyptienne et de l'Institut d'anatomie, dont le directeur Gustav Schwalbe ainsi que Spiegelberg certifient l'exactitude. Le document, daté des 8 et 11 juillet, s'intitule " Liste des factures à propos des frais de l'emballage et du transport des objets déterminés trouvés par le Professeur Dr. Spiegelberg à l'occasion des fouilles sur le site de la Thèbes égyptienne, pour la collection égyptologique et pour l'Institut d'anatomie ". Les dépenses couvrent les frais de douane pour le transport des caisses d'antiquités en Égypte, la traversée depuis Thèbes ouest jusqu'à Louqsor, l'acheminement jusqu'au Caire, le fret maritime jusqu'à Brême et enfin le trajet continental jusqu'à Strasbourg. Les caisses de la collection égyptienne sont entreposées pour étude au Jardin botanique, tandis que les trois autres sont dûment inventoriées à l'Institut d'anatomie. Sans jamais énoncer explicitement le contenu des malles, une note elliptique, attestant la légitimité des dépenses, laisse en réalité peu de place au doute : "Les trois malles sont utilisées pour l'objectif de l'Institut ». Elles contiennent donc évidemment des pièces anatomiques prélevées dans la nécropole thébaine, des parties de momies égyptiennes telles qu'on peut en voir encore aujourd'hui dans la collection de l'Institut d'anatomie normale à l'hôpital civil de Strasbourg ! L'intérêt des spécimens momifiés antiques pour les travaux de l'Institut est patent : anatomiste de grand renom, Gustav Schwalbe menait en effet ces années-là des recherches d'anthropologie physique sur des populations diverses dans le temps et dans l'espace (Zur Anthropologie der nordamerikanischen Indianer, 1897 ; Über die Schädelformen der ältesten Menschenrassen mit besonderer Berücksichtigung des Schädels von Eguisheim, 1897 ; Studien über Pithecanthropus erectus Dubois, 1899 ; Der Neanderthalschädel, 1901, etc.). Dans la foulée du Pithécanthrope, du Néandertal, du crâne d'Eguisheim ou des Indiens d'Amérique, quoi de plus naturel que de s'intéresser à la morphologie des anciens habitants de la vallée du Nil ? Schwalbe avait d'ailleurs ramené lui-même d'un voyage égyptien, en 1892, de nombreux crânes et têtes de momies, pour compléter la collection strasbourgeoise d'ossements représentatifs des populations humaines ${ }^{45}$ - le consul allemand à Louqsor, par exemple, lui avait ainsi remis 160 crânes contenus dans quatre sacs... ${ }^{46}$ Dans cette lignée, la livraison des trois malles de Spiegelberg, 


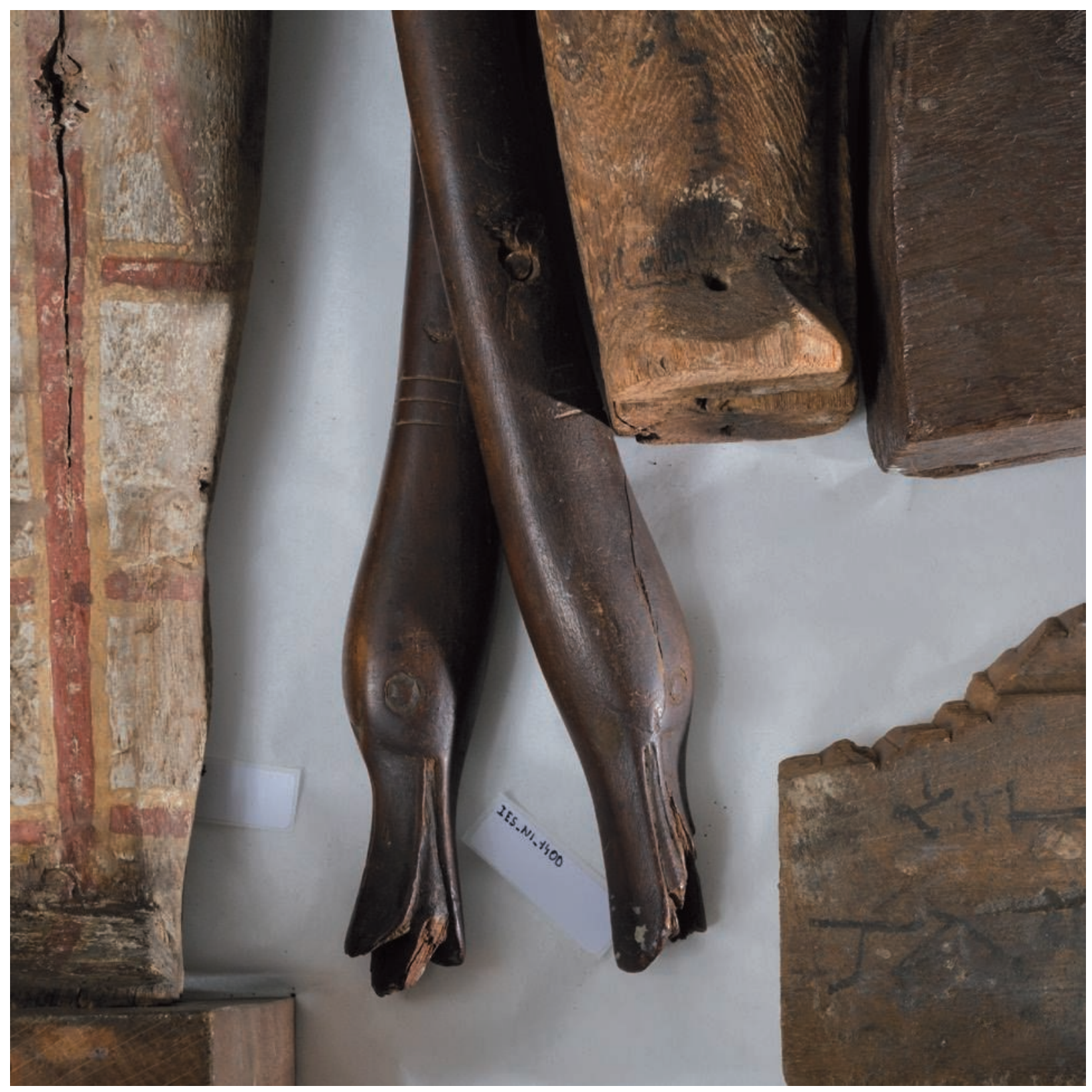

Pieds de chaise pliante décorés de têtes de canards, offerts par l'égyptologue belge Jean Capart (Musées royaux des arts décoratifs et industriels de Bruxelles) en échange de vases en terre cuite de l'ancien Empire achetés par Spiegelberg lors de l'expédition de 1895/96 (coll. UdS) 
sept ans plus tard, doit-elle s'interpréter comme une sorte de cadeau scientifique au milieu de la recherche médicale et anthropologique auquel appartenait son beau-père von Recklinghausen, en accompagnement des nouvelles sociabilités inaugurées par le mariage?

\section{Frédéric Colin}

\section{Notes :}

1 - A. Martin, P. Heilporn, " La collection de papyrus de la Bibliothèque nationale et universitaire de Strasbourg ", in W. Clarysse, H. Verreth (éd.), Papyrus Collections World Wide, 9-10 March 2000 (Bruxelles - Leuven), Bruxelles, 2000, p. 77-80

2 - P. Bucher, J. Leclant, « La collection de l'Institut d'égyptologie de l'Université de Strasbourg ", Bulletin de la société académique du Bas-Rhin pour le progrès des sciences, des lettres, des arts et de la vie économique, 75-78, 1953-1956, p. 100-109

3 - O. Primavesi, "Zur Geschichte des Deutschen Papyruskartells ", ZPE 114, 1996, p. 173-177 ; 180-183, Dok. 1-4

4 - J. Schwartz, Les archives de Sarapion et de ses fils, Le Caire, 1961 (BdÉ 29), p. 6

5 - ADBR AL 103154

6 - A. Erman, Mein Werden und mein Wirken. Erinnerungen eines alten Berliner Gelehrten, Leipzig, 1929, p. 169

7 - Straßburger Correspondenz, n. 131, 20 décembre 1897

8 - ADBR AL 103 862. Pour alléger les notes, les références au Journal de la Faculté de philosophie, dont les entrées datées dans l'ordre chronologique guident la suite de l'exposé, n'y seront pas systématiquement répétées.

9 - ADBR AL 103 873, 15 février 1896, requête de Michaelis au Staatssekretär du Ministère d'Alsace-Lorraine

10 - ADBR AL 103 873, 8 février 1896, requête de Michaelis au Kurator

11 - ADBR AL 103 873, 19 décembre 1896, requête de Michaelis au Kurator

12 - ADBR AL 103872

13 - ADBR AL 103 877, 21 mars 1898, requête de Reitzenstein et de Spiegelberg au Statthalter

14 - ADBR AL 103 877, 24 mars 1898, lettre de Zeppelin (bureau du Statthalter) au Ministère

15 - ADBR AL 103 877, 22 avril 1898, lettre du Staatssekretär à Reitzenstein

16 - Sans compter les honoraires des cours " privatim », c'est-à-dire payants pour les auditeurs.
17 - ADBR AL 103 680. Les documents officiels invalident ici la chronologie établie par Spiegelberg lui-même dans son curriculum vitae, conservé dans une note manuscrite $\left(1^{\text {er }}\right.$ octobre 1899 , cf. A. Grimm, Wilhelm Spiegelberg als Sammler, München, 1995, p. 1) ; le savant se référait probablement, de mémoire, au mois dans lequel était tombée sa première rentrée académique comme professeur, au semestre d'hiver.

18 - ADBR AL 103 873, 26 juin 1899, demande du curateur au doyen de la Faculté de philosophie

19 - Statut für die Kaiser-Wilhelms-Universität Straßburg vom 24. Februar 1875, Strasbourg, 1904, § 82-85

20 - ADBR AL 103 680, 2 août 1899, annonce du curateur au doyen de la Faculté ; AL 103 873, copie de la nomination du 27 juillet 1899

21 - ADBR AL 103 877, 10 mai 1899, rapport épistolaire de Reitzenstein et Spiegelberg au Statthalter

22 - Par exemple ABNU DPK A, pièce $n^{\circ} 6598$ (3/11/1899); compte de " Glasplattlieferungen " du 31/7/1899 au 9/3/1900 (sans n ${ }^{\circ}$; pièce $\mathrm{n}^{\circ} 2218(3 / 4 / 1900)$

23 - ABNU DPK A, pièce $\mathrm{n}^{\circ} 7751$ (21/12/1899)

24 - ADBR AL 103 862, Journal der philosophischen Facultät, entrée du 8 janvier 1900

25 - Rapport du 10 mai 1899, voir note 21

26 - ABNU DPK A, pièce $n^{\circ} 377$ (13/1/19oo)

27 - S. P. Vleeming, " Spiegelberg in Strasbourg ", Enchoria 11, 1982, p. 94

28 - O. Primavesi, "Zur Geschichte des Deutschen Papyruskartells ", p. 173, n. $7 ; 175$, n. 20

29 - L'abréviation " doc. ", dans le corps du texte, renvoie aux documents sur lesquels est fondée la présente synthèse. Document $1: 11$ juillet 1895, requête de Michaelis au Kurator (ADBR AL 103 873). Doc. 2 : projet non daté de Spiegelberg (ibidem), en accompagnement du doc. 1. Doc. 3 : 8 février 1896, requête de Michaelis au Kurator (ibidem). Doc. $4: 15$ février 1896 , requête de Michaelis au Staatssekretär (ibidem). Doc. 5 : 21 mars 1898, requête de Reitzenstein et de Spiegelberg au Statthalter (ADBR AL 103 877). Doc. $6: 13$ février 1899, requête de Michaelis au Kurator (ADBR AL 103 873). Doc. 7 : 10 mai 1899, rapport épistolaire de Reitzenstein et Spiegelberg au Statthalter (ADBR AL 103 877). L'édition commentée de ces textes, comme des principaux documents cités dans cet article, est en préparation.

$30-$ R. Reitzenstein, Zwei religionsgeschichtliche Fragen nach ungedruckten griechischen Texten der Strassburger Bibliothek, Strasbourg, 1901, p. V

31 - Fr. Igersheim, L'Alsace et ses historiens 1680-1914. La fabrique des monuments, Strasbourg, 2006, p. 256-259

32 - Sur la prééminence, aux yeux des philologues, des œuvres littéraires sur les textes documentaires au XIX ${ }^{\mathrm{e}}$ siècle, P. van Minnen, " The Origin and Future of Papyrology from Mommsen and Wilamowitz to the Present, from Altertumswissenschaft to Cultural Studies ", in Proceedings of the 2oth International Congress of Papyrology (23-29 August 1992), Copenhague (1994), p. 36-37. 
33 - A. Martin, O. Primavesi, L'Empédocle de Strasbourg (P. Strasb. gr. Inv. 1665-1666). Introduction, édition et commentaire, Strasbourg, Berlin, New York, 1999

34 - W. Spiegelberg, Zwei Beiträge zur Geschichte und Topographie der thebanischen Necropolis im Neuen Reich, Strasbourg, 1898, p. 2

35 - Ph. Derchain, « Débris du temple-reposoir d'Aménophis I $^{\text {er }}$ et d'Ahmes Nefertari à Dra Abou'l Naga ", Kêmi 19, 1969, p. 17-21

36 - Le fantôme d'Herculanum, et les fantasmes scientifiques qui l'accompagnaient, avaient déjà plané en 1892, lorsque Naville découvrit dans une ruine incendiée les archives carbonisées du nome Mendésien, sans pouvoir les exploiter efficacement ; H. Cuvigny, "The Finds of Papyri : the Archaeology of Papyrology ", in R. S. Bagnall (éd.), The Oxford Handbook of Papyrology, Oxford, 2009, p. 47.

37 - ADBR AL 103 877, 24 mars 1898, lettre de Zeppelin (bureau du Statthalter) au Ministère

38 - ADBR AL 103 877, 28 mars 1898, notice du Staatssekretär von Puttkamer

39 - E. Turner, Greek Papyri. An Introduction, Oxford, 1968 (1980), p. 22-24 ; J. G. Keenan, "The History of the Discipline ", in R. S. Bagnall (éd.), op. cit., p. 61

40 - W. Spiegelberg, Ägyptische und andere Graffiti (Inschriften und Zeichnungen) aus der thebanischen Nekropolis, Heidelberg, 1921, p. VI

41 - Journal de Percy Newberry, Lord Northampton's excavations 1898/9, consulté au Griffith Institute (Oxford), avec l'aimable autorisation du Pr. Jaromir Málek

42 - AMRAH, dossier " Capart objets 1903-1905 ", minute d'une lettre de Capart à van Overloop en réponse à son précédent courrier. Je remercie Valérie Montens, responsable des archives, pour son aide dans leur consultation.

43 - Brillamment soutenue par Ruey-Lin Chang, sous la direction des professeurs Jean Gascou et Andrea Jördens.

44 - ADBR AL 103873

45 - J.-M. Le Minor, " Gustav Schwalbe (1844-1916) ", in B. Schnitzler, J.-M. Le Minor, B. Ludes, E. Boës (éd.), Histoire(s) de squelettes. Archéologie, médecine et anthropologie en Alsace, Strasbourg, 2005, p. $295-297$

46 - G. Schwalbe, Reisebriefe aus Aegypten, Jena, 1893, p. 123, cf. p. $59-60 ; 103-104 ; 121 ; 135 ; 161-162 ; 174$

\section{ABRÉVIATIONS}

ABNU DPK A : Archives de la BNU, Deutsches Papyruskartell, Abteilung A

ADBR AL : Archives départementales du Bas-Rhin, Alsace-Lorraine

AMRAH : Archives des Musées royaux d'art et d'histoire (Bruxelles) 